San Jose State University

SJSU ScholarWorks

Master's Theses

Master's Theses and Graduate Research

Summer 2015

\title{
Clarifying the Role of Social Support in the Relationship between Stressors and Strains at Work: The Effect of Self-Construals
}

Maria Joao Subtil de Carvalho

San Jose State University

Follow this and additional works at: https://scholarworks.sjsu.edu/etd_theses

\section{Recommended Citation}

de Carvalho, Maria Joao Subtil, "Clarifying the Role of Social Support in the Relationship between Stressors and Strains at Work: The Effect of Self-Construals" (2015). Master's Theses. 4581.

DOI: https://doi.org/10.31979/etd.rfz2-59je

https://scholarworks.sjsu.edu/etd_theses/4581

This Thesis is brought to you for free and open access by the Master's Theses and Graduate Research at SJSU ScholarWorks. It has been accepted for inclusion in Master's Theses by an authorized administrator of SJSU ScholarWorks. For more information, please contact scholarworks@sjsu.edu. 
CLARIFYING THE ROLE OF SOCIAL SUPPORT IN THE RELATIONSHIP BETWEEN STRESSORS AND STRAINS AT WORK: THE EFFECT OF SELF-CONSTRUALS

\author{
A Thesis \\ Presented to \\ The Faculty of the Department of Psychology \\ San José State University \\ In Partial Fulfillment \\ of the Requirements for the Degree \\ Master of Science
}

by

Maria João S. de Carvalho

August 2015 
(C) 2015

Maria João S. de Carvalho

ALL RIGHTS RESERVED 
The Designated Thesis Committee Approves the Thesis Titled

CLARIFYING THE ROLE OF SOCIAL SUPPORT

IN THE RELATIONSHIP BETWEEN STRESSORS AND STRAINS AT WORK:

THE EFFECT OF SELF-CONSTRUALS

by

Maria João S. de Carvalho

APPROVED FOR THE DEPARTMENT OF PSYCHOLOGY

SAN JOSÉ STATE UNIVERSITY

$\begin{array}{ll} & \text { August } 2015 \\ & \\ \text { Dr. Sharon Glazer } & \text { Department of Psychology } \\ \text { Dr. Nancy Da Silva } & \text { School of Management } \\ \text { Dr. Jeremy Welland } & \text { Pandora Media, Inc. }\end{array}$




\section{ABSTRACT \\ CLARIFYING THE ROLE OF SOCIAL SUPPORT \\ IN THE RELATIONSHIP BETWEEN STRESSORS AND STRAINS AT WORK: THE EFFECT OF SELF-CONSTRUALS}

by Maria João S. de Carvalho

Practitioners and researchers often focus on social support to allay occupational stress, despite inconsistent results from empirical studies. This longitudinal study examined the impact of nurses' self-construals on the effect of social support on the relationship between stressors and outcomes, based on research showing that people in individualistic and collectivistic cultures perceive supervisor and coworker support differently and research showing culture is internalized as self-construals. The aim of the study was to identify circumstances in which social support mitigates stress. Survey data were collected at two points in time (about four weeks apart), from 139 nurses in two northern California hospitals, and subjected to hierarchical multiple regression and correlation analyses. Results showed that most models for direct effects of social support on outcomes were supported and none of the models for two-way interactions of support and stressors on outcomes were supported. However, self-construal did interact with social support and stressors but in the opposite direction of what was hypothesized. Coworker support aggravated the effect of role overload on anxiety for interdependents (reverse buffering) but not independents. Supervisor support aggravated the effect of role ambiguity on health and burnout for independents but mitigated it for interdependents (buffering). These findings, while opposing the hypotheses, still showed the importance of context in the role of social support on occupational stress and this role's complexity. 


\section{ACKNOWLEDGEMENTS}

I am extremely grateful for my wonderful thesis committee, Dr. Sharon Glazer, Dr. Nancy Da Silva, and Jeremy Welland, PhD. They brought a wealth of knowledge and experience to my thesis, as well as motivation and support to push me to the finish line, despite the length of time to get here; I always felt they had my success in mind. A special thank you to Dr. Sharon Glazer (thesis chair) for her expertise, the motivational check-ins, quick turnarounds, and overall availability for all my questions. Thank you Dr. Nancy Da Silva for the rigor, ongoing incentive, and for making me feel less guilty of passed deadlines. Thank you Jeremy Welland, $\mathrm{PhD}$, for the creative suggestions, questions that made me think, and for always simplifying. I also want to recognize Dr. Howard Tokunaga's constant availability and responsiveness, as well as technical advice.

This thesis is the culmination of a graduate school journey that I will always cherish. Thank you to my família (family) and amigos (friends) "back home" for their full support since the day I said I was going for a Masters abroad, especially my sister. Thank you to faculty, colleagues, and local family for making this journey a great one, and making me feel welcome from day one. I wholeheartedly dedicate this work to my awesome husband - Bruno - and super mother - Teresa, with endless gratitude for always pushing me to better myself and being pillars in my life. Bruno always made sure I had the availability to focus on this work, and I have no words to express how thankful I will always be for it. As I finalize these acknowledgments, I cannot help but think of my guardian angel, my father, who I always carry in my heart and who told me many years ago I could do anything I set my mind on doing... 


\section{TABLE OF CONTENTS}

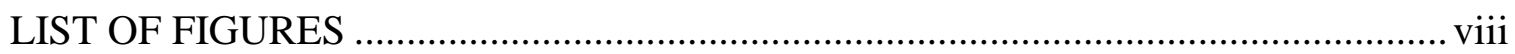

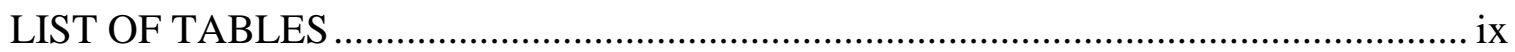

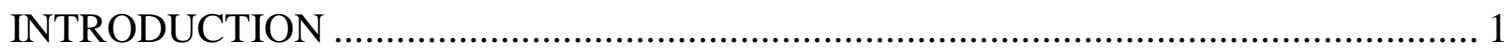

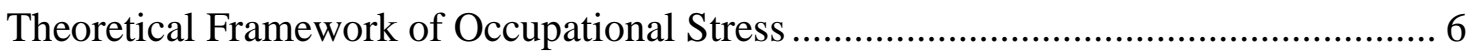

The Beehr and Newman's facet model..................................................................... 11

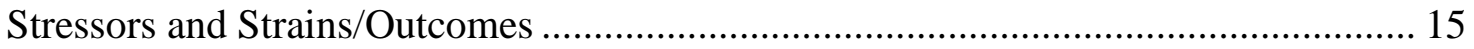

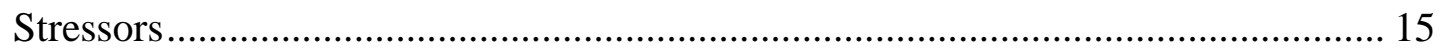

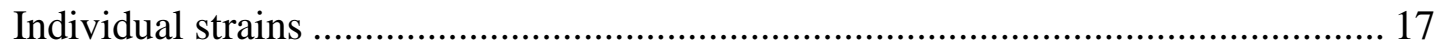

Organizational outcomes …………………………........................................ 19

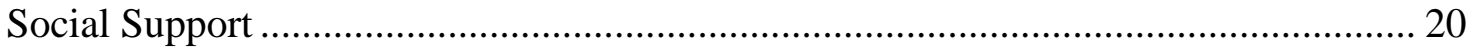

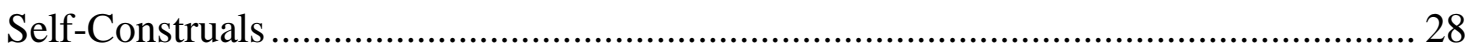

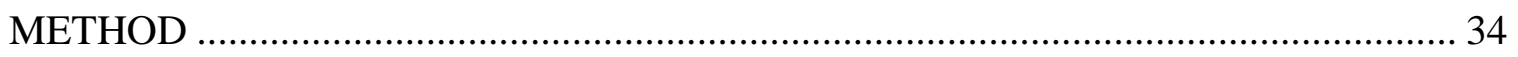

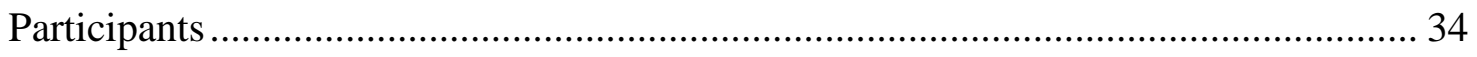

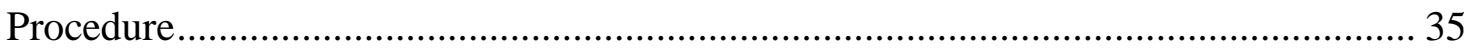

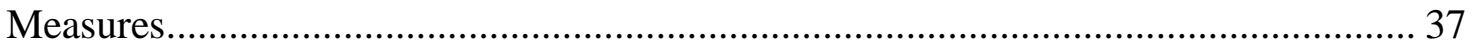

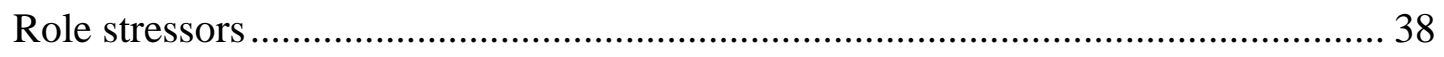

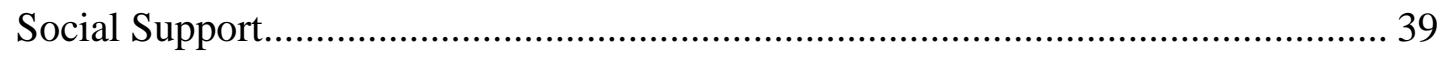

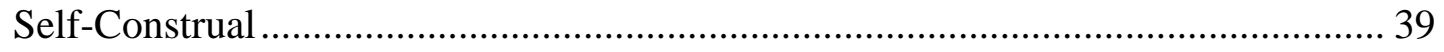




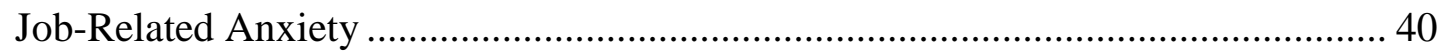

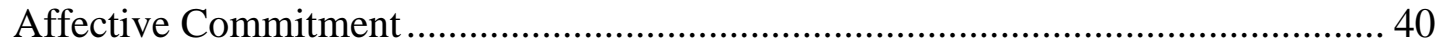

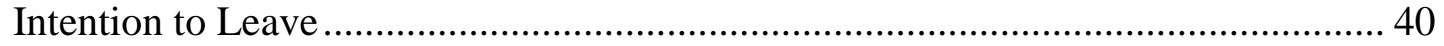

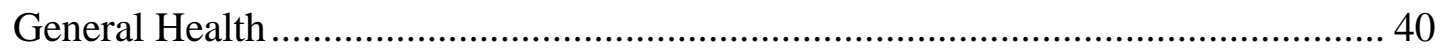

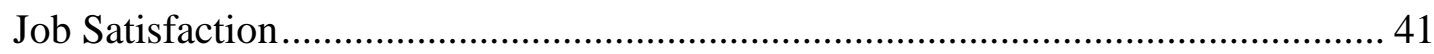

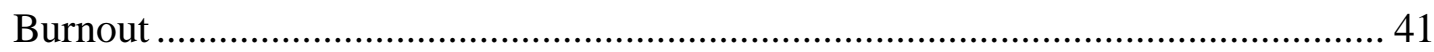

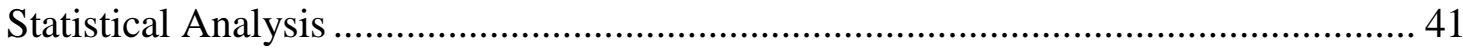

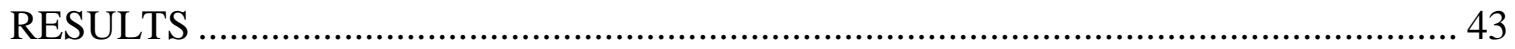

Correlations and Main Effects of Social Support on Strains/Outcomes ..................... 43

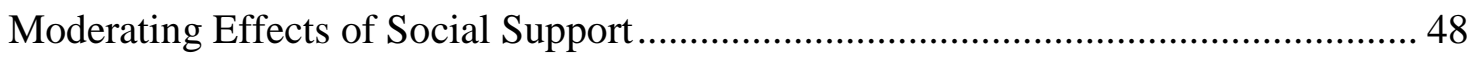

Moderating Effects of Social Support in Interaction with Self-Construal ................... 56

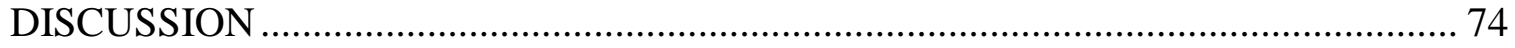

Limitations and Recommendations for Future Studies ....................................... 82

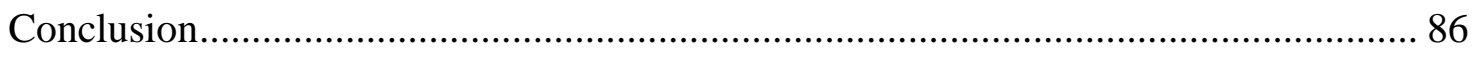

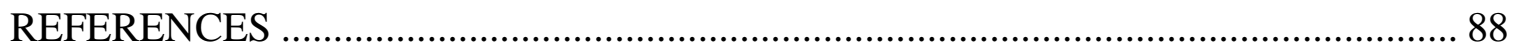

Appendix A - List of Items Used for Current Study ................................................ 97 


\section{LIST OF FIGURES}

Figure 1. Beehr and Newman's (1998) facet meta-model .......................................... 14

Figure 2. Theoretical framework for the current study and its focal variables............... 15

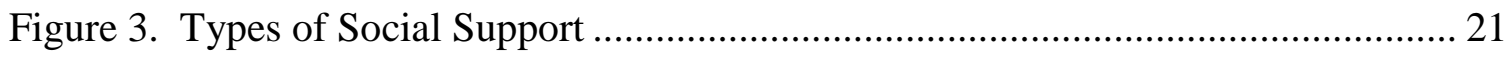

Figure 4. Models of Social Support tested in the current study.................................. 26

Figure 5. Three-way interaction hypotheses tested............................................... 33

Figure 6. Interaction between Role Overload, Coworker Social Support, and SelfConstrual on Anxiety........................................................................... 67

Figure 7. Interaction between Role Ambiguity, Supervisor Social Support, and SelfConstrual on General Health .................................................................... 70

Figure 8. Interaction between Role Ambiguity, Supervisor Social Support, and Self-

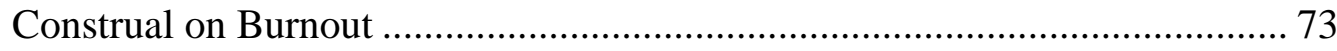




\section{LIST OF TABLES}

Table 1. Summary of Occupational Stress models presented........................................... 8

Table 2. Measures from each Survey Administration Utilized for the Current Study .... 38

Table 3. Means, Standard Deviations, Correlations, and Alpha Reliabilities (on Diagonal) Among Main Variables

Table 4. Factor Loadings for Principal Components Factor Analysis with Oblimin Rotation of Role Stressors

Table 5. Factor Loadings for Principal Components Factor Analysis with Oblimin Rotation of Supervisor Social Support

Table 6. Factor Loadings for Principal Components Factor Analysis with Oblimin Rotation of Coworker Social Support.

Table 7. Factor Loadings for Principal Components Factor Analysis with Oblimin Rotation of Anxiety 46

Table 8. Factor Loadings for Principal Components Factor Analysis with Oblimin Rotation of Affective Commitment

Table 9. Factor Loadings for Principal Components Factor Analysis with Oblimin Rotation of Intention to Leave.

Table 10. Factor Loadings for Principal Components Factor Analysis with Oblimin Rotation of General Health

Table 11. Factor Loadings for Principal Components Factor Analysis with Oblimin Rotation of Burnout

Table 12. Summary of Control Variables Used in Inferential Analyses (Regressions) for each Outcome

Table 13. Anxiety Regressed on Role Stressors, Social Supports, and their Interaction 51

Table 14. Affective Commitment Regressed on Role Stressors, Social Supports, and their Interaction.

Table 15. Intention to Leave Regressed on Role Stressors, Social Supports, and their Interaction

Table 16. General Health Regressed on Role Stressors, Social Supports, and their Interaction. 
Table 17. Job Satisfaction Regressed on Role Stressors, Social Supports, and their Interaction

Table 18. Burnout Regressed on Role Stressors, Social Supports, and their Interaction 56

Table 19. Anxiety Regressed on Role Stressors, Social Supports, Self-Construal, and their Interaction...

Table 20. Affective Commitment Regressed on Role Stressors, Social Supports, SelfConstrual, and their Interaction

Table 21. Intention to Leave Regressed on Role Stressors, Social Supports, SelfConstrual, and their Interaction

Table 22. General Health Regressed on Role Stressors, Social Supports, Self-Construal, and their Interaction

Table 23. Job Satisfaction Regressed on Role Stressors, Social Supports, Self-Construal, and their Interaction

Table 24. Burnout Regressed on Role Stressors, Social Supports, Self-Construal, and their Interaction.

Table 25. Anxiety Regressed on Role Overload, Coworker Social Support, SelfConstrual, and their Interaction

Table 26. General Health Regressed on Role Ambiguity, Supervisor Social Support, Self-Construal, and their Interaction

Table 27. Burnout Regressed on Role Ambiguity, Supervisor Social Support, SelfConstrual, and their Interaction 


\section{Introduction}

...People are disturbed not by things, but by their perception of things...

- Epictetus -

Occupational stress costs U.S. businesses nearly $\$ 300$ billion each year (American Institute of Stress, n.d.), with $\$ 50$ billion to $\$ 150$ billion attributed to health and productivity costs alone (HumanNature@Work, 2008); other costs include accidents, turnover, or workers' compensation claims and awards. Forty percent of job turnover is attributed to stress and so is $60-80 \%$ of workplace accidents (American Institute of Stress, n.d.). From the perspective of the worker, the 2014 Stress in America ${ }^{\mathrm{TM}}$ survey (American Psychological Association, 2015) showed, as prior editions did, that work was the second most named cause of strain (after money) for Americans, with $60 \%$ of people attributing their experience of strain to work.

Occupational stress surveys collected over the last two decades have shown an increase in workers reporting stress on the job and graver consequences as a result (American Institute of Stress, n.d.). The Stress in America ${ }^{\mathrm{TM}}$ survey for 2014 (American Psychological Association, 2015) reported that since 2007, stress levels decreased but were still considered far from healthy, especially for those who reported experiencing extreme stress. Additionally, $42 \%$ of Americans reported stress as a driver to unhealthy behaviors, and some groups reported higher levels of stress: women have consistently reported higher levels of stress than men since the onset of the survey, with an increasing gap between the two groups; Millennials and Gen Xers reported higher levels of stress than Boomers and Matures, and parents higher levels than non-parents. 
To rectify these debilitating results, company management and human resources departments have implemented interventions that include support networks within the workplace and overall promotion of social support (National Institute for Occupational Safety and Health, 1999; 2008), such as discussion of organizational practices through one-on-on mentoring or team meetings. Despite the promise these interventions are expected to hold, the impact of social support programs on occupational stress reduction has been inconsistent (Beehr, Bowling, \& Bennett, 2010; Beehr \& Glazer, 2001; Mayo, Sanchez, Pastor, \& Rodriguez, 2012; Nahum-Shani \& Bamberger, 2011). The additional factors that affect when social support moderates the stressor-strain relationship remain a mystery. One possible explanation is cultural context or aspects of the person's cultural background such as culture as construed in the mind of the individual. If in fact this influence exists, then nuanced interventions can be designed and implemented for higher efficacy in mitigating the deleterious effects of occupational stress.

According to Beehr and Glazer (2001), there appears to be some indication that different types and sources of social support moderate the stressor-strain relationship, depending on culture. In fact, Glazer (2006) found preference for social support does differ across cultures, with a preference for supervisory social support in individualism and for coworker social support in collectivism. However, whether or not this preference influences the extent to which social support moderates the stress-strain relationship was not tested. More recent studies (Morimoto, Shimada, \& Ozaki, 2013) showed, for example, that sociocultural beliefs play a role on how coping resources moderate stressor-strain relationship; although Morimoto and colleagues (2013) did not specifically 
study social support, social support is considered a possible ameliorative coping resource. By extending Beehr and Glazer's (2001) and Glazer's (2006) findings to the individual level, the present study investigated the role of independent and interdependent selfconstruals on social support's moderating effects on stressor-strain relationships.

The main purpose of this study was to contribute with clarity to the occupational stress field of research, by investigating how self-construals (Markus \& Kitayama, 1991) interact with the moderating effects of supervisory and coworker social support on occupational stress. Therefore, this study makes four contributions to the literature. First, it explores the possibility that the mitigating impact of social support in the relationship between stressors and strains goes beyond determining its existence or nonexistence, but rather it may be a matter of determining the circumstances in which that impact is stronger or weaker. Second, it is based on a meta-model of occupational stress, the Beehr and Newman facet model (Beehr, 1995; 1998; Beehr \& Newman, 1978), thus guaranteeing that it takes into account the most relevant theoretical conceptualization and research variables on the topic of occupational stress. Third, it focuses on culture as construed in the mind of the individual versus presuming a cultural orientation on the basis of group affiliation, and bringing to light the importance of situated meaning (Oyserman, 2011). Fourth, it uses a two-wave longitudinal survey research methodology, instead of a cross-sectional one, utilizing stressors from the first wave and outcomes from the second wave in the analyses.

Given the impact of occupational stress on the individual overall well-being, as well as on the economy, much research has unfolded over the course of the last five 
decades, starting with Kahn, Wolfe, Quinn, Snoek, and Rosenthal's (1964) seminal work on role stressors. Occupational stress is a complex construct and many approaches have been taken to define causes, consequences, and how best to interrupt, as well as, to mitigate it. Social support on the job as a way to mitigate (or buffer) the consequences of the occupational stress process has always had vast intuitive adherence to it, yet little research has lent clear support to this intuition. Weak, inconsistent or even reversed results have been attributed to lack of robust definitions, not considering different types and sources of social support, and the possibility that social support does mitigate occupational stress but under specific environmental circumstances or individual characteristics and not in a continuous, linear, fashion.

This study contributes to the body of research on the effects of social support on occupational stress by exploring the potential moderating factors in this relationship; this would result in a set of circumstances (individual and/or environmental) that would potentiate that effect and, conversely, another set under which there would be no effect, or even a reverse one. It is suggested in the present study that the very meaning of social support to any given person might vary depending on how they have construed their selfsystem (Markus \& Kitayama, 1991). Given that social support results, by definition, from the interaction with others, and given that self-construals refer to how one interprets the relationship with others and how distinct are the "self" and the "others," it was hypothesized that people with independent and interdependent selves will integrate and react to social support differently. Due to the different nature of coworkers' and supervisors' roles, it was expected people would react to social support differently 
depending on whether it comes from the supervisor or from coworkers. This study anticipated that supervisory social support would buffer occupational stress for people with independent selves, and coworker social support would buffer occupational stress for people with interdependent selves.

These hypotheses were tested on archival data gathered from nurses. Nursing is a high-stress profession (Tyler \& Cushway, 1995); the Bureau of Labor Statistics indicates that "registered nurses need emotional stability to cope with human suffering, emergencies, and other stresses" (Bureau of Labor Statistics, 2012). Moreover, O.Net documents "stress tolerance" as one work style associated with the job of a registered nurse, such that the "job requires accepting criticism and dealing calmly and effectively with high stress situations" (O.Net, 2010). The health industry, in general, has been characterized as one with the highest levels of reported occupational stress, namely by the Health and Safety Executive, the equivalent in Great Britain to the National Institute of Occupational Safety and Health in the USA (Health and Safety Executive, n.d.).

House (1981) asserts that understanding moderating processes of occupational stress is "especially significant because they offer the only feasible means of protecting and promoting health in situations where (further) stress reduction or health promotion are impossible or prohibitively expensive" (p. 6). The remainder of this introduction focuses on the theoretical framework that underlies this study, including a presentation of the model that governed this thesis, as well as of other influential models of occupational stress research. The main concepts and variables of this study are presented (stressors, 
outcomes, and social support and self-construals as moderators) along with the study hypotheses.

\section{Theoretical Framework of Occupational Stress}

Occupational stress is the general process by which aspects of the work (i.e., stressors) negatively affect the worker's physical and psychological health, often referred to as strains (Beehr, 1998; Beehr, Farmer, Glazer, Gudanowski, \& Nair, 2003; Jex, 2002). This is the stimulus-response definition (Jex, 2002), frequently used in the field, where stressors and strains are clear and distinct terms, and the term "stress" is used solely to refer to the process of the relationship between stressors and strains. This approach attempts to remove the lack of clarity in day-to-day references to "stress," meaning both the negative aspects of the work ("I have a lot of stress at my work") and the negative consequences of them ("I am feeling a lot of stress").

This study followed an organizational approach to occupational stress (Beehr, 1995). This approach to occupational stress is one of four approaches that both Beehr (1995) and Jex (2002) refer to as the main perspectives researchers have assumed when undertaking studies on this topic. The remaining three approaches are the medical approach, the clinical/counseling psychology approach, and the engineering psychology (human factors) approach.

More specifically, within the organizational psychology approach to occupational stress, this study was guided by a version of the Beehr and Newman facet model (Beehr, 1995; 1998; Beehr \& Newman, 1978). It is a meta-model in the sense that it was developed by analyzing the available theoretical models and research around 
occupational stress, and by proposing a model that would be overarching and fit most relevant theoretical conceptualizations and research variables on the topic (Beehr, 1998). Before Beehr and Newman's facet model is explored further, a brief overview of its most influential precursors in occupational stress research is presented. Table 1 contains a summary of these models.

The Institute for Social Research (ISR) model (Jex, 2002; Jex \& Beehr, 1991; Katz \& Kahn, 1978) was one of the earliest to be proposed and resulted from the research work conducted at the University of Michigan. This model starts with the worker examining the objective work environment, that is, the actual work-related events and conditions. Attention is then given to what is called psychological environment; this step of the model refers to the worker's perceptions of the previously referred objective environment. These perceptions are developed through a process of cognitive appraisal (i.e., judgment of the objective work environment) and, therefore, the psychological environment is subjective. Following this stage, instant reactions on the part of the worker will ensue, which can be of psychological, physical and/or behavioral nature. Finally, the model assumes that these immediate reactions will affect the mental and physical health of the worker. According to this model, these stages occur in a chain-ofevents type of process, such that previous steps precipitate following steps. Additionally, the ISR model considers that each and every relationship of one stage with the next is moderated by individual differences of the worker, both of a trait-like nature (e.g., personality, demographics, etc.) and interpersonal nature (e.g., support). 
Table 1

Summary of Occupational Stress Models Presented

\begin{tabular}{|c|c|}
\hline Model & Summary of Main Assertions \\
\hline $\begin{array}{l}\text { ISR Model } \\
\text { (Katz \& Kahn, } \\
\text { 1978) }\end{array}$ & $\begin{array}{l}\text { - The psychological work environment is developed out of the } \\
\text { objective work environment, through cognitive appraisal } \\
\text { - The worker reacts instantly to the psychological work } \\
\text { environment; these reactions impact physical and mental health } \\
\text { - Relationship between stages is moderated by trait-like and } \\
\text { interpersonal individual differences }\end{array}$ \\
\hline $\begin{array}{l}\text { McGrath's } \\
\text { Process Model } \\
\text { (McGrath, 1976) }\end{array}$ & $\begin{array}{l}\text { - An environmental situation is converted into a perceived situation } \\
\text { through cognitive appraisal. } \\
\text { - The worker acts out a selected behavior (performance process) } \\
\text { after going through a process of conscious decision-making } \\
\text { - The behavior will influence the initial situation characterizing this } \\
\text { process as a loop }\end{array}$ \\
\hline $\begin{array}{l}\text { Karasek’s Job } \\
\text { Strain Model/ } \\
\text { Demand-Control } \\
\text { Model } \\
\text { (Karasek, 1979) }\end{array}$ & $\begin{array}{l}\text { - Strains result of the combination of job demands and the worker's } \\
\text { degree of job control; this combination creates four outlooks: } \\
\text { passive jobs, low strain jobs, active jobs and high strain jobs } \\
\text { - Extensive research on it has added elements and increased scope } \\
\text { to an initial model otherwise described as less comprehensive }\end{array}$ \\
\hline $\begin{array}{l}\text { Person- } \\
\text { Environment Fit } \\
\text { model } \\
\text { (Edwards \& } \\
\text { Harrison, 1993; } \\
\text { French, Caplan, } \\
\text { \& Harrison, 1982; } \\
\text { Harrison, 1978) }\end{array}$ & $\begin{array}{l}\text { - Occupational stress depends on the degree of fit between the } \\
\text { worker and the work environment (less fit, greater strain) } \\
\text { - The model identifies two types of misfit between the worker and } \\
\text { the work environment: (a) between the supplies of the work } \\
\text { environment and the demands of the person, and (b) between the } \\
\text { demands of the environment and the abilities/skills of the worker } \\
\text { - Considers both subjective and objective aspects of the } \\
\text { environment and of the worker }\end{array}$ \\
\hline $\begin{array}{l}\text { Beehr and } \\
\text { Newman's facet } \\
\text { model } \\
\text { (Beehr, 1995; } \\
\text { 1998; Beehr \& } \\
\text { Newman, 1978) }\end{array}$ & $\begin{array}{l}\text { - Workplace stressors, situational characteristics (both constitute } \\
\text { environmental facet), and personal facet interact in what } \\
\text { constitutes the process facet where decisions are made; associated } \\
\text { reactions result in human and organizational consequences } \\
\text { - The model considers an adaptive response facet when } \\
\text { consequences create positive-type responses by the worker, and a } \\
\text { time facet acknowledging the temporal dimension in the model }\end{array}$ \\
\hline
\end{tabular}

Subsequent to the ISR model, others followed that looked at the stress process from different perspectives or highlighted different features of it. McGrath's (1976) 
process model encompasses four stages, conceptualized in a loop. This loop starts with the environmental situation (first stage), which then converts into perceived situation (second stage) through cognitive appraisal. A key distinctive feature of this model is added at this point; McGrath considered that before reacting, the person goes through a stage of selecting the reaction (third stage), which is determined by a process of conscious decision-making. Only after selecting his or her response will the worker act out that choice in a behavior (fourth stage) via what McGrath termed the performance process. The loop closes by linking behavior back to situation through the outcome process; this shows McGrath's consideration that the outcome will itself influence the situation that gave rise to it (Jex \& Beehr, 1991).

The Job Demand-Control (JDC) model, as is most commonly known, or the job strain model, as Karasek (1979) called it, defends that the extent to which a job causes strain is dependent upon two dimensions: the demands of the job (or a work situation), and the degree of control (i.e., decision latitude or decision-making freedom) the worker has within that job. Combining these two dimensions results in four different outlooks, assuming each of them can be either "high" or "low." As such, a job low in both demands and in individual control is named a "passive job," whereas one low in demands and high in control is called a "low strain job." When a job has high demands and control, that job is said to be an "active job." Low control and high demands jobs are designated "high strain" jobs - of the four, this combination is the most "stressful" for the employee. Compared to the previously discussed models, the JDC model is less comprehensive, as it has a smaller scope (Jex, 2002). Nevertheless, it has been a model 
with an extensive amount of research being dedicated to it (Jex, 2002; Toivanen, 2012; Tsutsumi, Kayaba, \& Ishikawa, 2011; Wu, Chi, Chen, Wang, \& Jin, 2010). Moreover, a portion of this research has complemented the model with additional aspects to be considered in the occupational stress model (Van Der Doef \& Maes, 1999).

The fourth and last model covered in this introductory review is the PersonEnvironment Fit model (French et al., 1982; Harrison, 1978). The model asserts that occupational stress occurs as a function of the fit (or lack thereof) between the worker and his or her work environment; the less fit, the greater the strains (Edwards \& Harrison, 1993; Jex, 2002; Sonnentag \& Frese, 2003). Despite the fact that this is a very intuitive idea, there is considerable complexity in the ways misfit between worker and work environment have been thought of as leading to strains. As such, this notion was elaborated further into two ways in which misfit can take place between the worker and the work environment (Edwards \& Harrison, 1993; Sonnentag \& Frese, 2003). One type of fit that has been considered and researched is the fit between the supplies of the work environment (including rewards) and the demands of the person (including needs and preferences). The other form of fit is that between the demands of the environment (including requirements) and the abilities and skills of the worker. Furthermore, Sonnentag and Frese (2003) referred to the distinction between objective and subjective environment, and objective and subjective person; strains would be the result of misfit between the subjective environment and the subjective person, which in turn would be influenced by objective environment and the objective person, respectively. 
The Beehr and Newman's facet model. The initial version of this model (Beehr \& Newman, 1978) consisted of seven facets: environmental facet, human consequences facet, personal facet, process facet, organizational consequences facet, adaptive responses facet, and time facet. The environmental facet refers to the group of aspects and characteristics of the work environment and, as such, this is the facet where all workplace aspects are included, from features of the work and job, to work-related relationships, to physical aspects of the workplace. The personal facet includes the individual stable characteristics of the worker; typically studied variables of this facet are, for example, demographics or personality-type variables. The environmental and the personal facet would then interact (as the person interacts with his or her work environment) in the process facet, such that appraisal processes by the worker would come into play and decisions are made as to the negativity or positivity of the environment. If negativity is perceived, the reactions that result from that perception can affect both the worker and the organization. The ones that affect the worker are part of the human consequences facet, whereas the ones that affect the organization are part of the organizational consequences facet. As such, the human and the organizational consequences facets include all of the strains and organizational outcomes that will be further explored ahead in this introduction. The adaptive response facet refers to any positive-type response (typically from the worker or the organization) that is set forth with the goal of counteracting or relieving stressors, strains or both (Beehr, 1998; Jex, 2002).

Finally, the time facet intends to represent the concept that all the other facets exist within a temporal dimension that needs to be taken into consideration. This meta- 
model considers time is present in multiple ways (Beehr, 1995); not only is it present in the sequencing of events and processes, but also in the duration of stressors (acute vs. chronic), or the short-term vs. long-terms strains, just to name a few. According to Beehr (1995; 1998), time is the "forgotten facet," as it is hardly ever considered in the research analyzed, but is of essence for both methodological and conceptual reasons. In the present study, the time facet was incorporated in the two-wave approach utilized, where stressors and social support were assessed at Time 1 and strains/outcomes and selfconstruals at Time 2.

If time is the forgotten facet, Beehr $(1995 ; 1998)$ considered process the "mysterious" one, as research and theory do not always address it, nor have made clear advancements in what specifically happens in that chain of events that starts with the stressors and results in strains; this stressor-strain fundamental relationship constitutes the sine qua non condition for the very existence of occupational stress and the BeehrNewman model calls it the core relationship. According to these authors, uncertainty is the most important aspect of the process facet, where others have defended decisionmaking processes (McGrath, 1976), cognitive appraisal processes (Lazarus \& Folkman, 1984) or others of physiological nature. Beehr (1998), based on the expectancy theory of motivation, proposed that uncertainty about the outcomes of one's efforts is a process that happens immediately in reaction to exposure to stressors and, in combination with the importance attributed to those outcomes and the duration of those uncertainties, potentiates the experience of strains. 
These aforementioned facets constitute different dimensions within the overall stress process that aggregate typically researched variables (Jex, 2002). All these facets and relationships were conceived as a meta-model and intended to cover most aspects that had historically, and should in the future, be taken into account when studying occupational stress. However, Beehr $(1995 ; 1998)$ also presented a more detailed and refined model, with the goal of emphasizing and reorganizing some aspects that more frequently guided research. Figure 1 represents the initial facet meta-model and the refined model, allowing the visualization of similarities and differences.

One of the main changes in the refined model was the distinction, within the environmental facet, between stressors (those environmental aspects that trigger the stress process and antecede strains) and situational characteristics (most frequently studied as environmental moderators). Conceptually distinguishing between stressors and moderators of environmental nature is particularly critical for this study, because moderator effects are specifically addressed, including environmental moderators (namely, social support). 


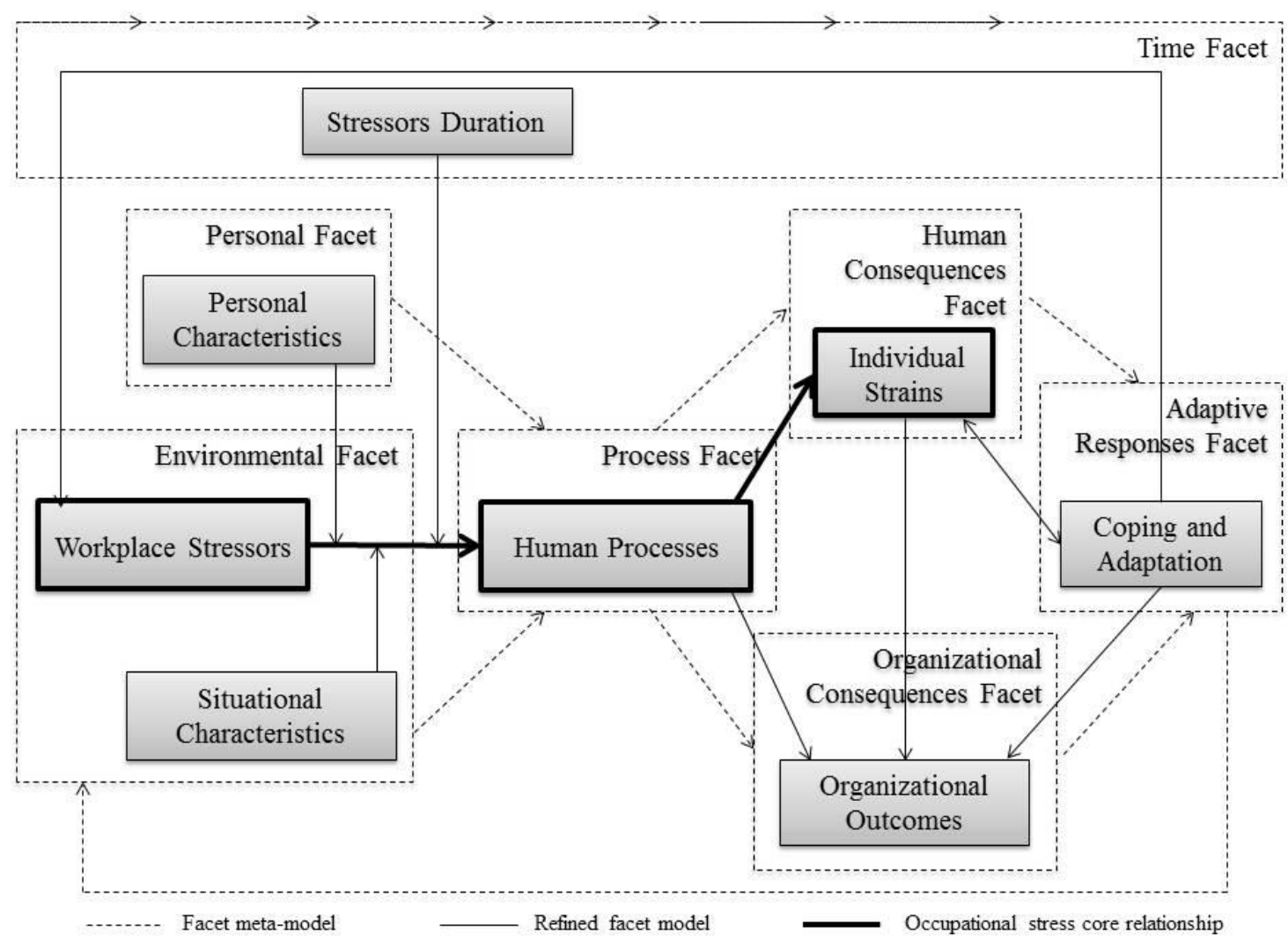

Figure 1. Beehr and Newman's (1998) facet meta-model. Adapted from Beehr (1998).

The specific way in which the current study followed this theoretical framework is depicted in Figure 2. This figure also shows the present study's focal variables, as they are associated with key theoretical concepts. As can be seen, this study included role overload, role conflict, and role ambiguity as workplace stressors, and six different outcomes/strains, namely anxiety, general health, burnout, and job satisfaction (individual outcomes), as well as affective commitment and intention to leave (organizational outcomes). Given that the goal of this study was determining the influence of social support and self-construals in occupational stress, supervisor and coworker social support were the situational aspects studied, whereas self-construals 
were the personal characteristics explored. The next section will explore these concepts and variables further, by focusing on stressors and strains, as well as organizational outcomes.

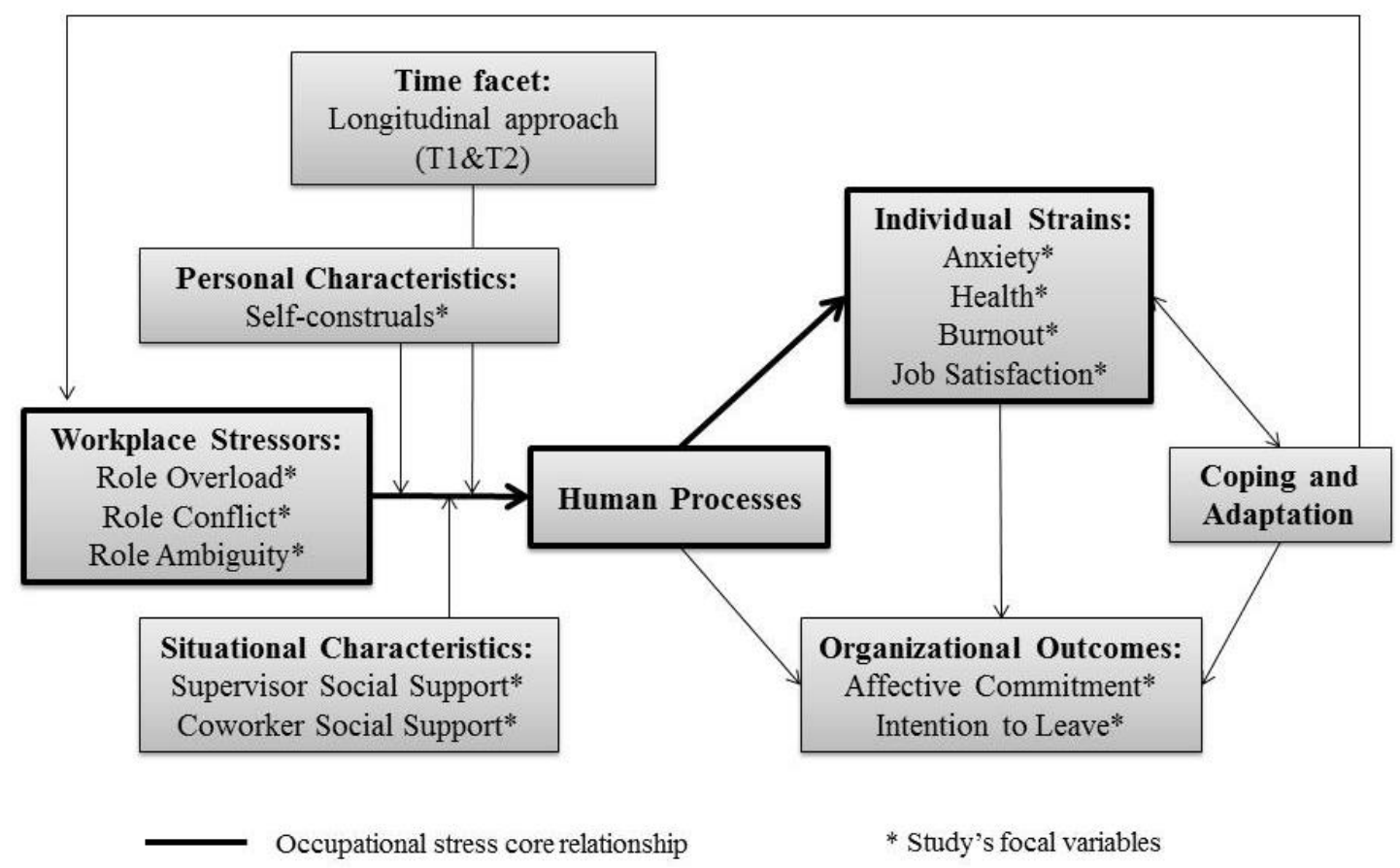

Figure 2. Theoretical framework for the current study and its focal variables. Adapted from Beehr (1998).

\section{Stressors and Strains/Outcomes}

Stressors. Within the study of occupational stress, stressors denote any event or characteristic of the work or workplace leading to adaptive reactions from the worker, which result in strain for him or her (Beehr, 1998; Beehr \& Glazer, 2001; Beehr et al., 2003; Jex, 2002). According to Jex (2002), most commonly studied job stressors are role stressors, workload, interpersonal conflict, organizational constraints (Peters \& O'Connor, 1980), and perceived control. Furthermore, Jex affirmed that work-family 
conflict, mergers and acquisitions, layoffs and job insecurity, as well as emotional labor, are less studied job stressors that are becoming more relevant due to recent trends. Role stressors are highlighted at this point because, as previously mentioned, they are the job stressors used in this study. Role stressors were chosen because most research on this topic has used role stressors (Beehr et al., 2010; Glazer \& Gyurak, 2008; Jex, 2002; Wu et al., 2010). With it, the goal was to refrain from adding more complexity to an already complex topic and align with the literature, with the novel contribution being the study of self-construals' influence on the occupational stress process.

Role stressors designate the group of job-related stressors that derive directly from the workers role set within the context of their job (Beehr \& Glazer, 2005; Glazer, 2005; Jex, 2002). More specifically, generic role stressors - and not job-specific stressors - will be addressed, as they reflect stressors associated with one's role that may arise in virtually any job. It is particularly relevant to study role stressors within the context of social support and occupational stress research because there is an intrinsic social component in role-associated stress; demands, expectations from others in the context of the workplace are at the core of why someone's role, and its associated script, can become stressors (Beehr \& Glazer, 2005).

Role ambiguity, role conflict, and role overload are three typically studied role stressors, with the last receiving somewhat less attention than the former two. Role ambiguity occurs when the worker is unclear about his or her job responsibilities, duties, and objectives, that is, what he or she should do (Glazer, 2005; Jex, 2002; King \& King, 
1990). An example can be the lack of definition on what one needs to do on the job in order to have the desired performance (Beehr \& Glazer, 2005).

Role conflict occurs when at least two sets of information or demands of the job present as incompatible, inhibiting the worker to be compliant with all the job demands at stake and be successful (Glazer, 2005; Jex, 2002; King \& King, 1990). This contradiction can present between two or more people or two or more roles of the worker's role set, in which case it is denominated inter-role conflict (Beehr \& Glazer, 2005). More specifically, if the contradictory expectations come from the same role sender, the conflict is called intra-sender; conversely, when different role senders are the source of conflict, it is said to be inter-sender role conflict. When the clash is between the expectations of the role and the worker's own values, it is named person-role conflict. This study focuses on inter-sender role conflict.

Role overload arises when additional demands to the job are to such extent that the worker cannot (or feels he or she cannot) perform everything that has been assigned to him or her (Glazer, 2005; Jex, 2002). According to Jex (2002), overload can refer to either the volume of work (quantitative role overload) and/or to the difficulty of the work that needs to be performed (qualitative role overload). The present study focused on quantitative role overload.

Individual strains. Stressors are aspects of the work that cause a reaction (outcome) on the workers that is negative to their well-being (in the broad sense of the word) or to the organization in which they work. These maladaptive reactions are named 
strains (Jex, 2002). Typically, strains can be categorized as psychological, physiological, and behavioral, depending on how they manifest.

Psychological strains, also called affective strains (Sonnentag \& Frese, 2003), are responses to stressors that are of a cognitive, emotional, or affective nature (Beehr \& Glazer, 2005; Jex, 2002). Examples are anxiety, general sense of well-being, mood disturbances, as well as attitudinal manifestations either toward the job or organizations or other life dimensions, such as job satisfaction and engagement. Eventually, upon continued exposure to stressors, mental health in general can be at risk as happens in the case of burnout, as well as depressive and psychosomatic symptoms.

Physiological strains refer to physical health and well-being consequences on the worker (Jex, 2002). According to Sonnentag and Frese (2003), hormonal, cardiac, and immunological reactions are some examples of physiological reactions an individual can manifest. Beehr and Glazer (2005) further added diabetes, weight fluctuations, rheumatic or orthopedic complaints, and gastrointestinal pain as examples of physiological strains. Moreover, in the long-term, chronic illness can result.

Finally, behavioral strains are the reactions that are "acted out" by the workers in response to stressors, in which they bring harm to themselves. Substance abuse (including alcohol or any type of drugs), accidents, counterproductive work behavior, and workplace violence are examples of behavioral strains (Beehr \& Glazer, 2005; Jex, 2002; Kivimäki, Hotopf, \& Henderson, 2010).

All of the individual outcomes studied in this research (anxiety, general health, burnout, and job satisfaction), and depicted in Figure 2, are of psychological nature. The 
present study treated General Health as a measure of general psychological well-being consistent with the research literature - rather than a measure of physical outcome (Banks, et al., 1980; Goldberg \& Hillier, 1979; Goldberg, et al., 1997). Individual psychological strains seem to develop more the more role-related job stressors are present, however this is not so much the case with physiological or behavioral strains (Beehr \& Glazer, 2005; Jex \& Beehr, 1991). Thus, the latter were not included in this study. Higher anxiety and burnout, as well as, lower general health and job satisfaction are expected along with higher experiences of role stressors.

Organizational outcomes. Other negatively charged attitudes, cognitions, and behaviors can be held by the workers as a consequence of occupational stressors. However, because they can potentially have a greater negative effect on the organization than on the worker, they are not considered a strain per se, but rather are classified in occupational stress literature as organizational outcomes (Beehr \& Glazer, 2005). As with individual strains, organizational outcomes are sub-categorized as psychological or behavioral.

Psychological organizational outcomes encompass the psychological responses of workers that are often antecedents of behaviors, which in turn are harmful to organizations (Beehr \& Glazer, 2005). Examples are psychological contract, organizational justice, intention to leave, and organizational commitment, with the last two being the most frequently researched outcome when the stressors at stake are role stressors. They are both expected to be related with role stressors (Beehr \& Glazer, 2005). Behavioral organizational outcomes are the actual behaviors carried out by the 
worker that affect the organizations negatively (Beehr \& Glazer, 2005). Examples are performance, absenteeism, turnover, grievances, and accidents. However, most often research has not revealed strong (or most of the time moderate, for that matter) or unequivocal relationships between role stressors and these organizational outcomes; therefore, they were not included in this study. This study assessed affective organizational commitment and intention to leave (otherwise known in literature as turnover intention) as organizational outcomes that are psychological in nature.

\section{Social Support}

The goal of this section is to review the concepts and state of research around social support as a potential moderator to the influence of occupational stressors on strains. Before discussing the role of social support in the occupational stress process, it imposes social support be defined and characterized. This is a challenging task as many definitions have been provided (Beehr, 1995; House, 1981). As with the word "stress," the expression "social support" is familiar to virtually everyone (Beehr, 1995; House, 1981) but, at the same time, as many definitions would likely come up as people being asked to define it.

Social support is a concept that encompasses many others and that may be the reason for the multitude of definitions. It can be thought of as a meta-construct by including other concepts within its scope (Beehr, 1995). The best way that several authors thought of to encompass the complexity of social support and, at the same time, realistically address its multifaceted nature, was to define types of social support and also to characterize it in terms of sources of social support. Nonetheless, general overarching 
definitions are provided and they relate with the broad notion that social support refers to the ways in which people assist or help one another in the numerous dimensions of life (work, personal, etc.), formally or informally (Beehr, 1995; Beehr \& Glazer, 2001; Munro, Rodwell, \& Harding, 1998). House (1981) illustrates this helpful component of social support by referring to a study resulting in 26 different ways in which respondents described being helped by someone else in dealing with a problem. The focus of the present study is on social support within the context of the work setting, and of social interactions with someone either in a supervisory or coworker role.

Regarding types of social support, the most used taxonomies are the ones distinguishing between structural and functional support, and between emotional (nontangible) and instrumental (tangible) support, with the latter being often times conceptualized as sub-taxonomy of functional support (Beehr, 1995; Beehr \& Glazer, 2001) as per Figure 3.

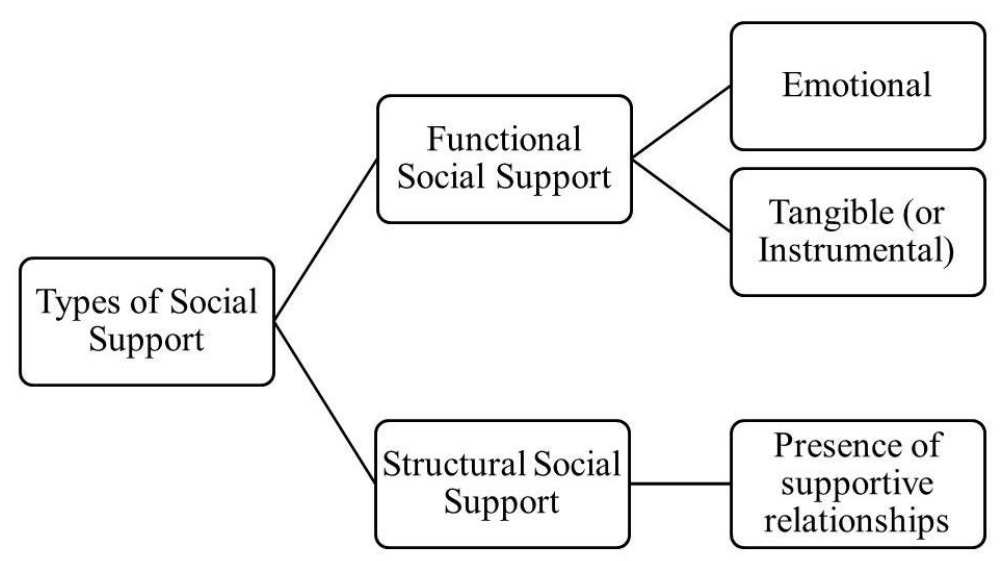

Figure 3. Types of Social Support. Adapted from Beehr and Glazer (2001). 
In referring to structural support, Cohen and Wills (1985) defined it as the mere existence of supporting relationships. As such, the multiple social structures to which one belongs would be one's structural support. Family (nuclear and extended), work teams, school, a sports team, or a religious group, are examples among the numerous social structures of which one might be a part (Beehr \& Glazer, 2001). Key elements or dimensions of social structures, particularly with respect to work-type of social structures, are size of the structure (i.e., number of people), density (the extent to which the structure is perceived by the focal person as psychologically or physically crowded), and shape (e.g., organizational hierarchy; Beehr \& Glazer, 2001). The distinctive feature about structural support is that it does not relate to the kinds of supportive actions taken toward the focal person; rather, it relates to the fact that supportive others exist, in the context of a social structure. As such, it might be viewed as more of a quantitative aspect of social support.

Functional social support, in contrast, might be interpreted as more of a qualitative aspect of a supportive relationship; the support serves a purpose to the focal person (Beehr, 1995; Beehr \& Glazer, 2001; Cohen \& Wills, 1985). These functions will be of a positive, helpful, tone and the overarching feature is that they ought to make the focal person's life easier; in the context of occupational stress, it would be their work life. This could be achieved by functional support, such as providing necessary information to the focal person, or giving them praise or positive feedback, so as to promote one's selfesteem, among other functions. As noted by Beehr and Glazer (2001), the fact the functional social support holds the monopoly of occupational stress research over 
structural social support probably relates to this heterogeneity of functional support; structural support would be far more consistent because the worker would necessarily have structural support just by belonging to an organization. Granted that Cohen and Wills (1985) proposed this taxonomy from the context of general stress research and not specifically of occupational stress research.

The two main functions that are considered within occupational stress research and literature are emotional support and tangible (or instrumental) support (Beehr, 1995; Beehr \& Glazer, 2001). Caplan, Cobb, French, Harrison, and Pinneau (1975) proposed this categorization and exemplified tangible support as help given through medical services and financial aid, and exemplified emotional support as help given through affection, understanding, or friendship.

Emotional support encompasses actions from supportive others that function to promote emotions and feelings of positive nature, and associates with the idea most probably comes to mind when thinking of social support (Beehr, 1995; Beehr \& Glazer, 2001). The core function here is not to help the focal person by acting upon the stressful situation or facilitating its resolution, but to help with the focal person's emotions toward the situation.

Tangible (or instrumental) support refers to any action by the supportive other that has a tangible result in helping solve a situation that is stressful to the focal person (Beehr, 1995; Beehr \& Glazer, 2001). This can be achieved by means of the supportive agent directly acting on the situation, (e.g., when a coworker helps another with their workload, or a supervisor extends a deadline), or by supplying the person the necessary 
resources for him or her to solve the situation themselves. These resources can be physical (e.g., tools), financial, informational or of any tangible, concrete, nature toward alleviating the focal person's problem (Beehr, 1995; Beehr \& Glazer, 2001). House (1981) additionally referred to informational support (providing information) and appraisal support (providing self-evaluation types of information), and admitted it was a challenge distinguishing these from other types of support and even between the two. For the purpose of this study these will be considered within instrumental social support.

Some issues with measuring both types of social support have been identified that may account for the inconsistent results on the moderating influence of social support (Beehr, 1995; Beehr \& Glazer, 2001). Regarding instrumental social support the issue relies on feasibility; that is, the extent to which the necessary conditions for a supportive other to help the focal person are present. As far as emotional support is concerned, one special issue that has been studied is oral communication, particularly the nature of what people tell each other and whether or not some aspects are particularly effective in mitigating the effects of stressors on strains (Beehr, 1995; Beehr \& Glazer, 2001; Beehr, Jex, Stacy, \& Murray, 2000; Beehr, King, \& King, 1990). An additional interesting issue within the functional social support research is the extent to which emotional and tangible support are separate or correlated, despite serving different functions (Beehr \& Glazer, 2001; Semmer, et al., 2008), and how general questionnaire measures are. However, the goal of this study was not to test how different types of social support yield different results in the moderating effect of social support on the relationship between stressors and strains. 
The sources of social support play an important role in characterizing it. House (1981) referred to nine different sources of social support; yet, from the perspective of occupational stress research, typically studied sources of social support fall within three classifications: social support from the supervisor, from the coworker, and from outsiders of the workplace context (Beehr \& Glazer, 2001). It is within the scope of this study to assess the different moderator effects for supervisor and coworker social support, given different self-construals. Support from others outside of the workplace context will be out of the scope of this study. Supervisory social support is that which is originated from people in the focal person's leadership, and most often studies inquire specifically about the direct supervisor. Social support from coworkers is, as the name clearly indicates, that which stems from peers, individually or in group.

Researchers have advanced multiple explanations for how social support impacts the relationship between stressors and strains (Beehr \& Glazer, 2001; O'Driscoll \& Dewe, 2001; Viswesvaran, Sanchez, \& Fisher, 1999); the models that represent these different considerations explored in this study are depicted in Figure 4. The direct effects models (components 1 in Figure 4) postulate that social support has a direct influence on strains and on stressors. That is, and for the idea of direct influence on strains, social support can directly alleviate strains the focal person may be experiencing, regardless of variation in stressors. Less researched, but also considered, is the possibility that social support would correlate with stressors, even though it is postulated that this relation could take many forms (Viswesvaran et al., 1999). 


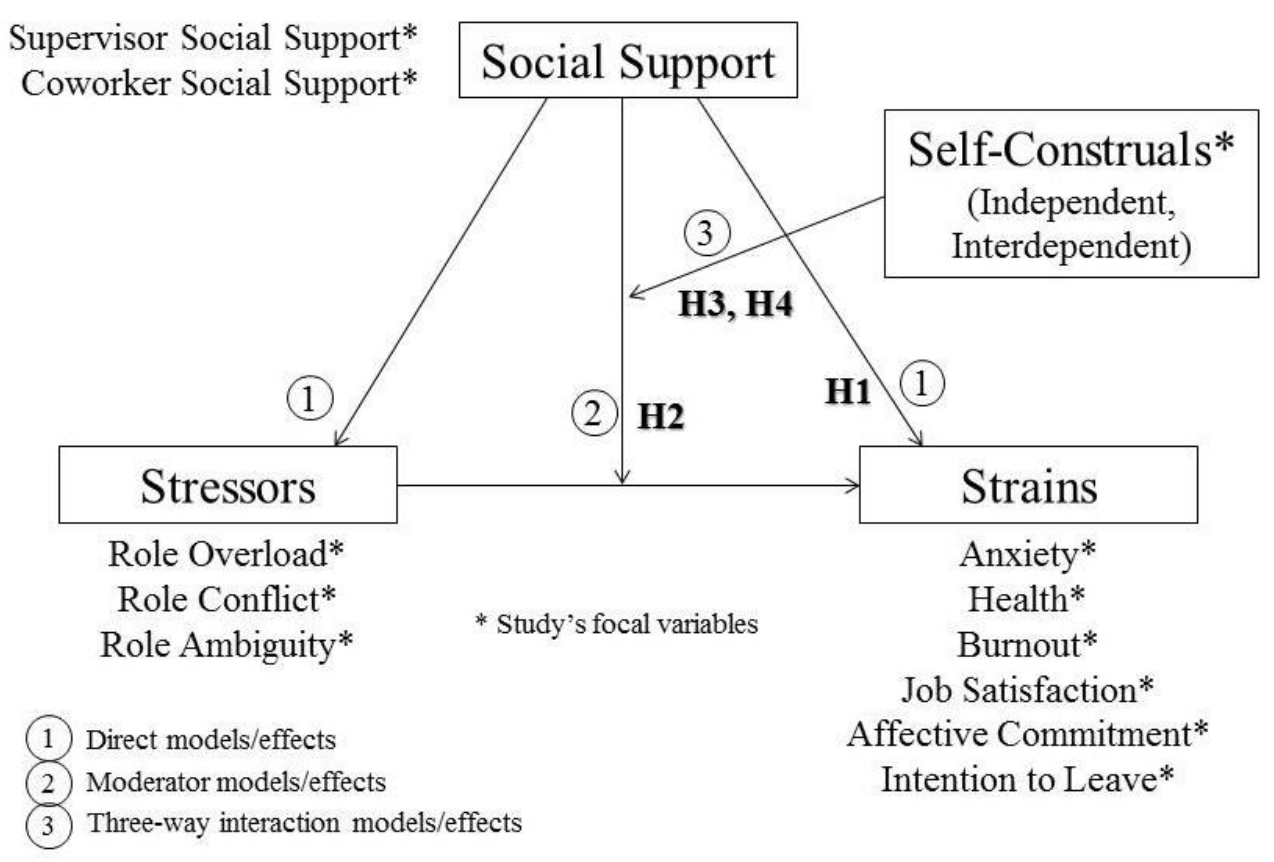

Figure 4. Models of Social Support tested in the current study.

Moderator models (component 2 in Figure 4) assert that social support explains when stressors relate to strains. More specifically, focus is on the buffering model, which suggests that for people with low social support, the relationship between stressors and strains will be stronger, which means an increased experience of strain. Conversely, people who perceive higher levels of social support are anticipated to experience less strain. Moderator models were the main focus of the current study.

Overall, the most consistent information about the influence of social support on occupational stress is that results on the moderator model have been inconsistent (and, at times, conflicting with what had been anticipated), and that main direct effects of social support on certain strains gather far more research support (Beehr \& Glazer, 2001; Ganster, Fusilier, \& Mayes, 1986; Viswesvaran et al., 1999). One of the challenges in 
accumulating research-based knowledge around the buffering effect of social support on the stress process is that no one consistent conceptual and operational definition allows for comparison among studies (Beehr, 1995; LaRocco, House, \& French, 1980).

Researchers have proposed other methodological explanations for the inconsistent results, including measurement selection, definition, and sampling issues (Beehr, 1995; Ganster et al., 1986).

Conceptually, theorists have suggested that the way social support has been thought of as relating with occupational stress might be one that needs a new conceptual approach, namely that the relationship might not be constant (Beehr, 1995; Kaufmann \& Beehr, 1986). In fact, interactions with other characteristics of the individuals or of the environment are possibilities that need to be considered (Beehr, 1995; Kaufmann \& Beehr, 1986). In practical terms, this means that the influence of social support on occupational stress, above and beyond direct effects, might vary depending on the person and the situation at stake, which would statistically necessitate a three-way interaction. This study explored this possibility by testing the extent to which self-construals can clarify how supervisory social support and coworker social support distinctively moderate the relationship between stressors and strains, as depicted in component three of Figure 4. To start, direct effects and simple moderator effect hypotheses were tested. $H_{1}$ : Social support (coworker and supervisor) will negatively correlate with unfavorable outcomes (lower anxiety, burnout, and intention to leave) and positively correlate with favorable outcomes (greater well-being, job satisfaction, and affective commitment). 
$H_{2}$ : The stressor-outcome relationship will be moderated by social support (coworker and supervisor) such that the relation between stressors and outcomes will be stronger in the presence of low social support, than in the presence of high social support.

\section{Self-Construals}

One of the possible explanations that have been advanced for the lack of clarity in results is that the effect of social support is itself moderated by other aspects (Beehr, 1995; Ganster et al., 1986). This study examined the impact of an individual's selfconstrual (Markus \& Kitayama, 1991; Singelis, 1994) on the effectiveness of social support received at work from either one's supervisor or coworker. Glazer (2006) demonstrated that people in individualistic and collectivistic cultures perceived supervisory and coworker support differently, after it had been theorized by Beehr and Glazer (2001) that there would be a relationship between cultural values (Schwartz, 1999) and social support from the supervisor and coworkers. As an extension of these findings to the individual level, the present study looked at the role of independent and interdependent self-construals exert on social support.

According to Singelis (1994), self-construal refers to "a constellation of thoughts, feelings, and actions concerning one's relationship to others, and the self as distinct from

others.” (p. 581). Despite this universal notion that a self-system is integral to everyone, individuals develop and construe their self-system differently and culture plays a substantial role in that divergence (Markus \& Kitayama, 1991; 2010; Nezlek, Kafetsios, \& Smith, 2008; Singelis, 1994). With that in mind, Markus and Kitayama (1991; 2010) proposed the concepts of independent and interdependent self-construals to capture how 
people would construe their beliefs regarding how connected or separate they are with others, and the impact of the self-construals in psychological processes (e.g., cognitive, emotional and motivational), as well as social processes.

The independent construal of the self (Markus \& Kitayama, 1991; 2010) refers to a representation of the self that is organized in function of one's own unique attributes such as cognitions, behaviors, motivations or emotions rather than those of other people. At the cultural level, the independent self is said to be promoted by the individualistic normative imperative (as exemplified by Western cultures), such that the norm of becoming independent and expressing one's uniqueness is that which becomes salient for people with a highly developed independent self-construal (Markus \& Kitayama, 1991; 2010; Singelis, 1994; Triandis, 1996). Other characteristics attributed to independent relationship preferences are direct communication and promotion of the individual's goals. These are not to say a person with a strong independent self-construal is alienated from their social environment; rather, these individuals are essentially autonomous, with social interaction focused on the uniqueness and internal attributes of those involved, and guided by, or toward, the realization and direct expression of those attributes. With inner characteristics taking the central role, the independent self is considered a "bounded whole" because those characteristics will be relatively stable.

The interdependent construal of the self is characterized more as a flexible one because connectedness with external aspects, such as relationships, roles, and statuses, as well as others' behaviors, cognitions, emotions, and motivations, takes central role (Markus \& Kitayama, 1991; 2010; Singelis, 1994). As all of these aspects change 
depending on the interactions and the situations, so will the behaviors of individuals with a highly developed interdependent self-construal. This is due to the driving forces of belongingness, appropriateness to the situations, and fundamentally fitting in. Indirect communication is attributed to those preferring interdependent relationships; by being constantly aware of one's external environment, the person who has an interdependent self-construal reads and assumes what others may think, feel or need, in accordance to the situation. In contrast with what is described for the independent self-construal, the cultural-level imperative that construes the interdependent self is the maintenance of the collective and the interdependence of those in the group, as is portrayed in the collectivistic normative (Fischer et al., 2009; Triandis, 1996). All these characteristics associated with the interdependent self, do not mean people highly developed in it do not have their own goals or govern their own actions, nor does it mean that people cannot function without others. Markus and Kitayama (1991) highlighted that the interdependent view does require considerable self-control to modify one's own behavior depending on the situation (which is interpreted as maturity and not loss of identity, as it may be interpreted by an independent view). Furthermore, they pointed out that for an interdependent perspective, the group or others' goals might be seen as personal or contributing to them might bring the individual the same level of satisfaction an independent self-construal would get from self-realization. In fact, where people with a highly developed independent self-construal see relationships as a way to achieve their unique potential, people with a highly developed interdependent self-construal take relationships as end goals in and of itself. The concept of an interdependent self- 
construal is, however, distinct from the concept of need for affiliation (McClelland, 1961; 1988). Markus and Kitayama (1991), in their exploration of consequences of independent and interdependent views of the self, posited that need for affiliation is a motivation which could, potentially, be more salient to an interdependent self-construal and not as much to an independent self-construal. As such, these would remain clearly distinct constructs to the extent that how people see themselves is different than what they are driven toward.

The current study suggests that the fact people do not all engage in, and interpret, social interactions (as a supportive interaction from a coworker or supervisor) the same way, may be the key to comprehending the unexpected results of social support role in the occupational stress research; with it, understanding the role of social support in the occupational stress process and the best way to leverage it in the workplace may be a step closer.

At the cultural level, Beehr and Glazer (2001) theorized the relationship between cultural values (Schwartz, 1999) and social support from the supervisor and coworkers, part of which Glazer (2006) later demonstrated. She found that across cultures, people perceive social support differently depending on whether supportive others are the supervisor or coworkers, such that "people in Autonomous cultures reported greater supervisor emotional support and less co-worker instrumental support than people in Conservative cultures" (p. 605). Schwartz's typology of cultural values aligns with that of Hofstede (2001, as cited in Glazer, 2006) such that Autonomy relates to Individualism, and Conservatism relates to Collectivism (Beehr \& Glazer, 2001; Glazer, 2006). 
If culture is in the mind of the person (represented by self-construals at the individual level due to the independent self-construal/individualism and interdependent self-construal/collectivism parallels), and if culture is believed to affect how social support moderates the stressor-strain relationship, it is expected, by extension, that these individual-level cultural constructs (i.e., self-construals) would act similarly. With supervisory social support being compatible with individualism and coworker social support being compatible with collectivism at the cultural level, it is expected that, at the individual level, supervisory social support will be compatible with independent selfconstruals and coworker social support will be compatible with interdependent selfconstruals. These assertions form the foundation for the following two hypotheses (also depicted in Figure 5).

$H_{3}$ : There will be a three-way interaction of coworker social support, role stressors, and self-construal in predicting outcomes, such that for individuals high on interdependent self-construal the relationship between role stressors and outcomes will be weaker for individuals with high coworker social support than low coworker social support.

$H_{4}$ : There will be a three-way interaction of supervisor social support, role stressors, and self-construal in predicting outcomes, such that for individuals high on independent self-construal the relationship between role stressors and outcome will be weaker for individuals with high supervisor social support than low supervisor social support. 

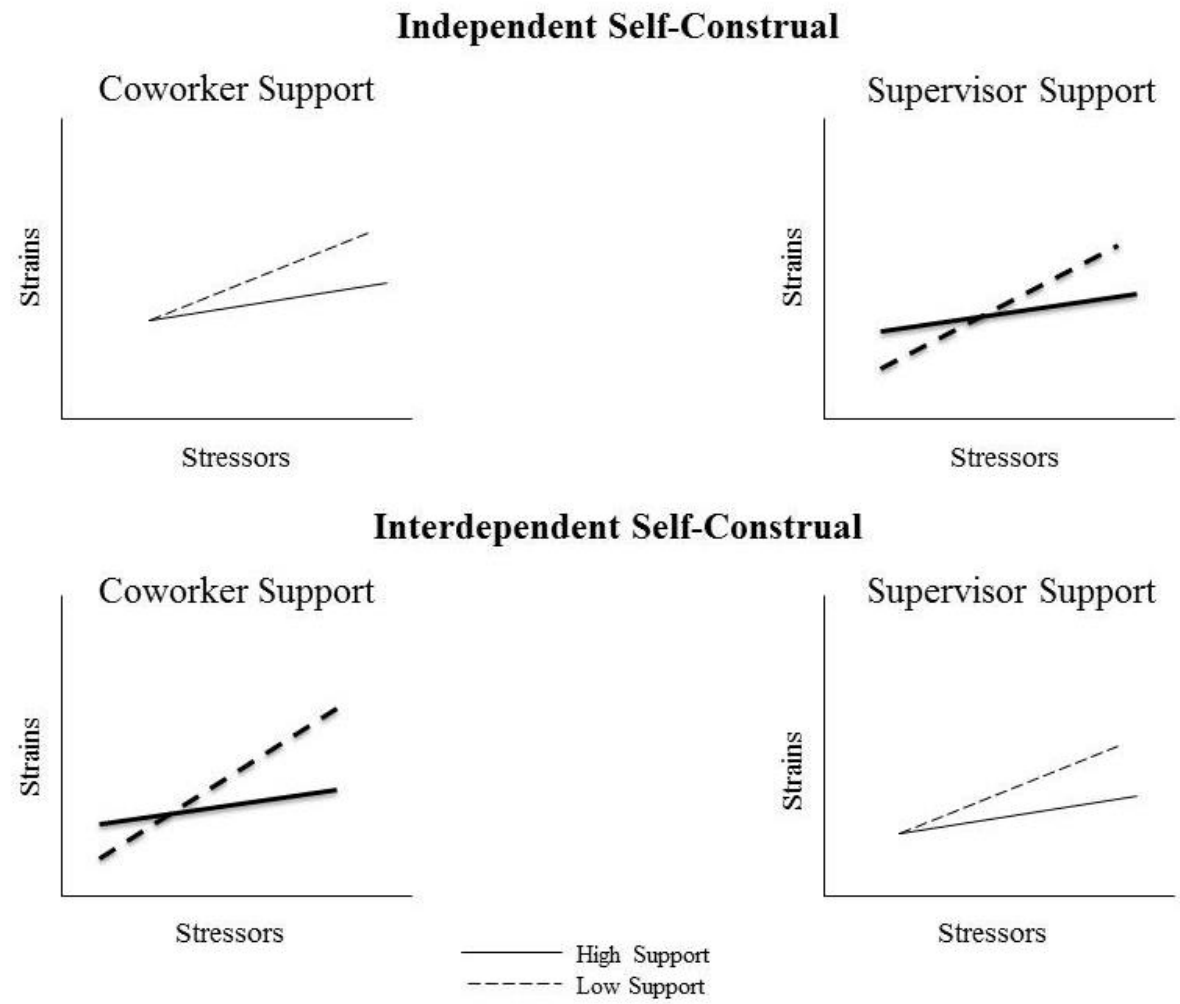

Figure 5. Three-way interaction hypotheses tested. 


\section{Method}

Data used in the current study derived from a larger research program on stress and leadership among nurses across cultures. Thus, data used for this study are archival.

\section{Participants}

Participants were registered nurses, as well as licensed nurses, working at two hospitals, in northern California, United States. For both hospitals, the study involved a two-time administration of different surveys. Although 304 nurses responded to the Time 1 survey and 225 responded to the Time 2 survey, the focus of analyses will be on the subset of data provided by 139 nurses from both hospitals who completed both surveys. These 139 respondents represent $36 \%$ of the 390 unique responses to either Time 1 or Time 2 surveys. More specifically, 83 nurses from Hospital 1 responded to Time 1 and Time 2 surveys (an 11\% response rate from the total 744 distributed surveys), and 56 nurses from Hospital 2 responded to both surveys (a 6\% response rate of the totally 990 distributed surveys).

The ages of the 139 respondents whose data were considered for this study varied from 27 to 71 years, with a mean of $50.33(\mathrm{SD}=9.98)$ years. Ninety-four percent of participants were female. Fifty nine percent of the nurses were White, $18.7 \%$ were Asian, 8.6\% were of other ethnicities, and $13.7 \%$ did not respond. Regarding all respondents at Time 1 ( $n=304$; when demographic questions were gathered), ages varied from 24 to 71 years old, with a mean of $47.54(\mathrm{SD}=10.28)$. Ninety-six percent of participants were female. Of the Time 1 participants, $50.3 \%$ were White, $23.4 \%$ Asian, $7.9 \%$ were of other ethnicities, and $18.4 \%$ did not respond. In comparison, of all the 
Time 2 participants $(n=225), 93 \%$ of those who responded were female. These figures suggest gender variations are not a concern for the final matched sample.

Additionally, the sample's demographic characterization was compared to the annual California Survey of Registered Nurses by the California Board of Registered Nursing (Spetz, Keane, \& Herrera, 2011), to confirm representativeness of the sample in face of low response rates. The survey's 2010 edition revealed that in the Bay Area region, most registered nurses with active California licenses were in the 50-54 years-old age group (15.1\%), followed by the 55-59 (13\%), 35-39 (12.3\%), 60-64 (11.8\%), and 4549 years-old (11\%) groups. Gender-wise, the survey pointed to $89.3 \%$ currently working nurses residing in California being female; results are not segmented by region.

However, information on gender of active registered nurses by age group is provided, showing variation between $88 \%$ (in the under-35-years group) and $96.3 \%$ female nurses (in the 65-years-and-older age group). Regarding ethnic and racial composition, the survey indicates the Bay Area region is typically composed of 54.6\% White nonHispanic, 19.8\% Filipino, 9.8\% Asian non-Filipino, 5.0\% Hispanic, 3.9\% Black/AfricanAmerican nurses, with $4.1 \%$ being of other ethnicities/races, and $2.8 \%$ not responding.

\section{Procedure}

Surveys were distributed to all registered and licensed nurses employed at the participating hospitals (about 750 nurses at Hospital 1 and about 1050 nurses at Hospital 2). Prior to survey administration, the Chief Nursing Officers sent an email to all nursing staff informing of the research program and the survey administration. In addition, the program's Principal Investigator presented its rationale and purpose to the nursing 
leadership team. The research team allowed two weeks for the completion and return of the first survey and the Time 2 survey was distributed about three weeks after Time 1 distribution (Dormann \& Zapf, 1999; Zapf, Dormann, \& Frese, 1996). An additional three weeks were permitted for the return of the completed second survey. Mid-way through each survey administration, the researchers posted reminders in each hospital unit.

Paper-pencil surveys were administered by placing surveys in each nurse's office mailbox or folder, distributing individually to each nurse, asking managers to distribute to their team (including themselves), and through internal mailing. Once distributed, nurses could take surveys home with them to complete. Nurses returned surveys in sealed envelopes to the Chief Nursing Offices, addressed to the program's Principal Investigator.

As a motivator to complete the survey and a token of gratitude for completing it, all nurses received a ticket to a local theater (each a $\$ 10$ value donated to the Principal Investigator's research program) along with the first survey, regardless of whether or not they completed the survey. Additionally, as an incentive to completing both surveys, 16 gifts (valued between $\$ 80$ and $\$ 200$ ) were raffled in each hospital after completion of the two surveys. Examples of gifts were: Nintendo Wii ${ }^{\mathrm{TM}}$ Console, Kodak EasyShare ${ }^{\mathrm{TM}}$ Digital Camera, and Garmin ${ }^{\mathrm{TM}}$ GPS System, among others. To participate in the raffle, nurses had to mark that they were interested in doing so by indicating it in the survey. All nurses received two raffle tickets along with the Time 2 survey. One raffle ticket was to be returned with the completed Time 2 survey and the nurse retained the other. 
All data were collected anonymously, without any obvious identifying information being collected. However, in order to match Time 1 and Time 2 surveys and at the same time guarantee participants' anonymity, participants were asked to create a self-generated identification code (guided by questions and instructions; Damrosch, 1986;

DiIorio, Soet, Van Marter, Woodring, \& Dudley, 2000; Yurek, Vasey, \& Havens, 2008). The self-generated identification code questions are presented in Appendix A.

\section{Measures}

Time 1 and Time 2 surveys from the larger research program contained mostly different measures (for a summary of items used in this study, see Appendix A). That is, there were measures assessed at Time 1 , others at Time 2 , and others were assessed at both Time 1 and Time 2. Data on stressors were gathered during both Time 1 and Time 2; nonetheless, as stressors are predictor variables, the present study utilized the Time 1 stressor data. Social support data were also gathered at Time 1. Given that the focus is on the interactions of stressors with social support, stressors and social support data needed to be gathered simultaneously. Outcome variables (including strains) were collected at Time 1 and Time 2 but more measures were administered at Time 2; therefore, this study utilized the Time 2 strain data. Additionally, self-construal was gathered at Time 2. Because self-construal is a considerably stable measure (Cross, Hardin, \& Gercek-Swing, 2011; Markus \& Kitayama, 1991), timing of its administration was theoretically inconsequential. All stressor, strain, and social support items, with exception of burnout, were rated on a 7-point Likert-type scale, ranging from 1 (strongly 
disagree) to 7 (strongly agree). Table 2 lists the measures utilized in this study.

Following there is a more detailed description of the measures.

Table 2

Measures from each Survey Administration Utilized for the Current Study

\begin{tabular}{ll}
\hline Time 1 measures & Time 2 measures \\
\hline Job-related stressors (10 items) & Self-Construal (7 items) \\
Social support (8 items) & Job-Related Anxiety (4 items) \\
& Affective Commitment (8 items) \\
& Intention to Leave (3 items) \\
& General Health (well-being, 11 items) \\
& Job Satisfaction (1 item) \\
& Burnout (10 items) \\
\hline
\end{tabular}

Role stressors. This study included three job-related role stressors (items 1-10 in Appendix A): role overload, role conflict, and role ambiguity, assessed through 10 items, at Time 1 administration. Three items (e.g., "I do things that are apt to be accepted by one person and not accepted by other") were used to assess role conflict, three items (e.g., "I have clear, planned goals and objectives for my job") measured role ambiguity, and four items (e.g., "I often notice a marked increase in my work load") captured role overload (Abdel-Halim, 1978; Beehr, Walsh, \& Taber, 1976; Rizzo, House, \& Lirtzman, 1970). Although the original measure contained five items for each role stressor, the Principal Investigator for the larger study chose to retain a subset in order to shorten the survey. The items were chosen on the basis of a multi-country factor analysis (Glazer \& Beehr, 2005). Items for role ambiguity were reverse coded. Cronbach's alpha 
reliabilities were .89 for the role overload scale (see items 1-4), .75 for the role conflict scale (see items 5-7), and .87 for the role ambiguity scale (see items $8-10$ ).

Social Support (see items 11-18). An eight-item scale modified (from question to statement format) from Caplan et al. (1975) was used at Time 1 to assess perceived coworker and supervisor social support. Four items referred to perceptions of supervisor support and the other four items referred to perceptions of co-worker support. An example supervisor support item is: "It is easy to talk with my supervisor at work" and an example coworker support item is "I can rely upon other people at work when things get tough at work." Cronbach's alpha reliabilities were .93 for the supervisor support scale (see items 11-14) and .89 for the coworker support scale (see items 15-18).

Self-Construal (see items 19-25). Self-construal (or relationship preference) was assessed through seven items from Goldstein (2008). For each item, participants chose between two statements presented side-by-side. On the left side were items indicative of an interdependent self-construal, whereas on the right side were items descriptive of an independent self-construal. An example item is: "It is important that my behavior is appropriate for the situation vs. It is important that my behavior and attitudes correspond." Respondents marked their preferred item from each pair. Interdependent self-construal items were scored as 0 and independent self-construal items were scored as 1. These scores were averaged so that a score closer to or 1 reflected greater tendency toward independent self-construal, whereas a score closer to or 0 reflected greater tendency toward interdependent self-construal. 
Job-Related Anxiety (see items 26-29). Four items were adopted from Parker and DeCotiis (1983) measure of job stress; these are four of the five items that constitute the anxiety dimension. Example items are "I have felt fidgety or nervous as a result of my job" and "My job gets to me more than it should." Cronbach's alpha reliability for this scale was .90 .

Affective Commitment (see items 30-37). Eight items were adopted from Allen and Meyer's (1990) measure of organizational commitment; these eight items represent the entire affective commitment dimension. An example of an item is "This organization has a great deal of personal meaning for me." Cronbach alpha reliability for this measure was .81 .

Intention to Leave (see items 38-40). Intention to leave was measured with three items adapted from the Michigan Organizational Assessment Questionnaire (Cammann, Fichman, Jenkins, Jr., \& Klesh, 1983). Like in Glazer and Beehr (2005), the first item was modified from the form of a question, asking about one's likelihood of looking for a new job, to a statement that follows the item structure of the other two items. An example item is "I often think about quitting." Reliability for this scale was .87 .

General Health (see items 41-51). General psychological distress was collected through a 12-item version of the General Health Questionnaire developed by Goldberg (Banks, et al., 1980; Goldberg \& Hillier, 1979; Goldberg, et al., 1997). Eleven items were retained for this study, which were modified for consistency with other items in that section, by rewording them into "I" statements. The 12th item was eliminated from this questionnaire because it used the word "strain," which can be problematic in the context 
of occupational stress research (Jex, Beehr, \& Roberts, 1992). An example of an item is: "I have lost much sleep over worry." Cronbach's alpha reliability for this scale was .84.

Job Satisfaction. Job Satisfaction was measured with a single item adapted from the Michigan Organizational Assessment Questionnaire (Cammann et al., 1983), "Overall, I am satisfied working at this organization." There is literature suggesting that a single item for job satisfaction is as robust and appropriate as using full measures (Wanous \& Reichers, 1996; Wanous, Reichers, \& Hudy, 1997).

Burnout (see items 53-62). A 10-item measure on burnout was adopted from Malach-Pines (2005). The measure asked participants "When you think about your work overall, how often do you feel the following?" and then listed the 10 items (e.g., "tired," "hopeless," and "physically weal/sick") that were rated on a scale ranging from 1 (“never") to 7 (“always"). Cronbach's alpha reliability for this scale was .88.

\section{Statistical Analysis}

Initially, an internal consistency analysis was conducted to test the main variables' reliabilities. Only for job satisfaction (single-item measure) and self-construal (a dichotomous ordinal measure) was it not possible to conduct this analysis. Then, principal components factor analyses were employed to validate the theorized constructs. For all dependent variables, as well as for the two social support variables, principal components analyses with oblimin-rotation were conducted assuming unidimensionality (i.e., testing for one factor). In addition, all role stressor items were subjected to a threefactor model (forced factor analysis), representing role overload, role conflict, and role ambiguity. 
From this point on, only previously computed composite variables were used. To assess the assumption of normality necessary for the subsequent analyses, graphical analyses were conducted and, despite slight deviations, it can be assumed that the variables present in this study follow a normal distribution. Next, descriptive statistics (means and standard deviations) were calculated as well as correlations among all main variables. Correlation analyses were also used to test Hypothesis 1.

Prior to conducting the inferential statistics that would allow testing the research hypothesis, available demographics variables were compared with this study's outcome variables. The goal was to determine which demographic variables might need to be used as control variables for each dependent variable while running inferential analysis. Correlations were conducted to establish the relationship with the quantitative demographics variables (age, number of children, professional tenure, organizational tenure, tenure in unit, and salary range; collected at Time 1) and ANOVAs to establish the relationship with the categorical demographic variables (hospital, whether or not the participant was born in the USA, ethnicity, marital status, employment status, job purpose, job title, and shift work pattern).

Finally, hierarchical moderated multiple regression analyses were performed to test Hypotheses 2, 3, and 4. To test Hypothesis 2, the six dependent variables were each regressed on role stressors and social support (supervisor and coworker). To test hypotheses 3 and 4, the six outcomes were each regressed on role stressors, social support variables, and self-construal. Significant three-way interactions were graphically analyzed to understand the nature and direction of the relationships. 


\section{Results}

Means, standard deviations, correlations, and Cronbach alpha coefficients (on diagonal) are presented in Table 3. In all instances of the principal components analyses with oblimin-rotation, factor loadings and total variance explained were at satisfactory levels to assume these measures behaved as intended. Factor loadings ranged from .32 to .96 (see Table 4 through Table 11).

\section{Correlations and Main Effects of Social Support on Strains/Outcomes}

Correlation analyses (see Table 3) were performed to support established relationships (e.g., between stressors and strains). All role stressors significantly correlated with all outcome variables, ranging from $|.24|$ to $|.43|$, and all of them in the expected direction; positive significant correlations were found with the unfavorable outcomes (strains: anxiety, intention to leave, and burnout), and negative significant correlations were found with the favorable outcomes (affective commitment, general health, and job satisfaction). A correlation analysis was also employed to test the Hypothesis $1\left(H_{1}\right)$, that supervisor and coworker social support will be associated with higher levels favorable outcomes and lower levels of unfavorable outcomes. Per Table 3, $H_{1}$ was supported. Coworker support positively correlated with affective commitment ( $r$

$=.35, p<.01)$, general health $(r=.29, p<.01)$, and job satisfaction $(r=.18, p<.05)$, and negatively correlated with anxiety $(r=-.23, p<.01)$, and burnout $(r=-.29, p<.01)$. Supervisor social support positively correlated with affective commitment $(r=.39, p<$ $.01)$ and job satisfaction $(r=.33, p<.01)$, and negatively correlated with anxiety $(r=-$ $.18, p<.05)$ and intention to leave $(r=-.28, p<.01)$. 
Table 3

Means, Standard Deviations, Correlations, and Alpha Reliabilities (on Diagonal) Among Main Variables

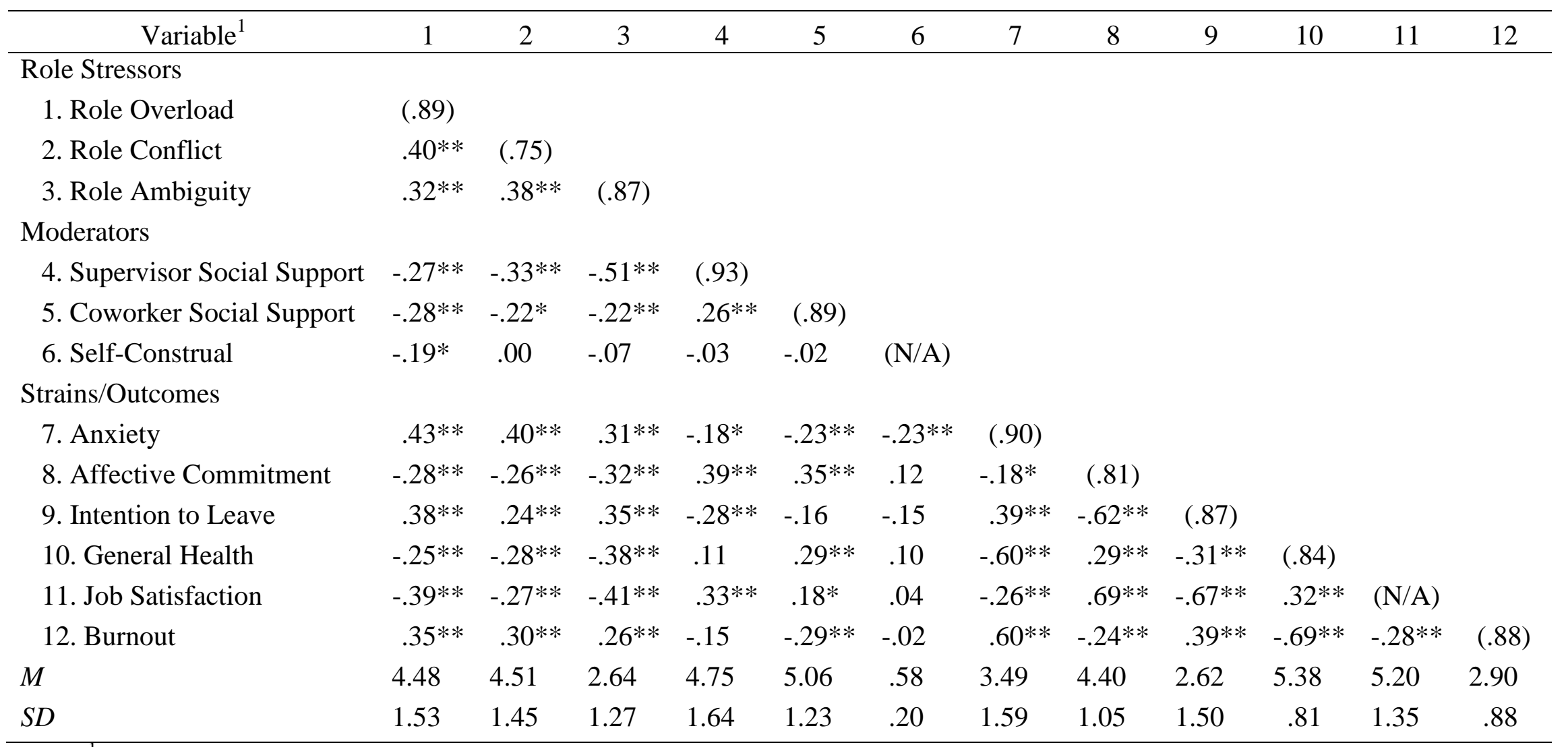

Note. ${ }^{1}$ Listwise $n=136$

$* p<.05$. ** $p<.01$. 
Table 4

Factor Loadings for Principal Components Factor Analysis with Oblimin Rotation of Role Stressors

\begin{tabular}{|c|c|c|c|}
\hline Item & $\mathrm{RO}$ & RA & $\mathrm{RC}$ \\
\hline It seems like I have too much work for one person to do. & .94 & & \\
\hline I often notice a marked increase in my workload. & .87 & & \\
\hline $\begin{array}{l}\text { On my present job, the amount of work seems to interfere with } \\
\text { how well I can do the job. }\end{array}$ & .87 & & \\
\hline I receive an assignment without the manpower to complete it. & .79 & & \\
\hline I know exactly what is expected of me. (R) & & .96 & \\
\hline I have clear, planned goals and objectives for my job. (R) & & .88 & \\
\hline Explanation is clear of what has to be done. $(\mathrm{R})$ & & .82 & \\
\hline $\begin{array}{l}\text { I do things that are apt to be accepted by one person and not } \\
\text { accepted by another. }\end{array}$ & & & .81 \\
\hline I work with two or more groups who operate quite differently. & & & .80 \\
\hline I receive incompatible requests from two or more people. & & & .76 \\
\hline \multicolumn{4}{|c|}{$\begin{array}{l}\text { Note. Factor loadings }>.30 \text { are presented. } \mathrm{RO}=\text { Role Overload, RA = Role Ambiguity, } \\
\text { and } \mathrm{RC}=\text { Role Conflict. }\end{array}$} \\
\hline \multicolumn{4}{|l|}{ Table 5} \\
\hline \multicolumn{4}{|c|}{$\begin{array}{l}\text { Factor Loadings for Principal Components Factor Analysis with Oblimin Rotation of } \\
\text { Supervisor Social Support }\end{array}$} \\
\hline Item & \multicolumn{3}{|c|}{$\begin{array}{l}\text { Supervisor } \\
\text { Social Support }\end{array}$} \\
\hline $\begin{array}{l}\text { My supervisor at work goes out of his or her way to do things to } \\
\text { make my work life easier for me. }\end{array}$ & \multicolumn{3}{|c|}{.94} \\
\hline It is easy to talk with my supervisor at work. & \multicolumn{3}{|c|}{.93} \\
\hline I can rely on my supervisor at work when things get tough at work. & \multicolumn{3}{|c|}{.90} \\
\hline My supervisor at work is willing to listen to my personal problems. & \multicolumn{3}{|c|}{.88} \\
\hline
\end{tabular}


Table 6

Factor Loadings for Principal Components Factor Analysis with Oblimin Rotation of Coworker Social Support

\begin{tabular}{lc}
\hline \multicolumn{1}{c}{ Item } & $\begin{array}{c}\text { Coworker } \\
\text { Social Support }\end{array}$ \\
\hline I can rely upon other people at work when things get tough at work. & .89 \\
It is easy to talk with other people at work. & .87 \\
Other people at work go out of their way to do things to make my work & .87 \\
$\quad$ life easier for me. & .83 \\
Other people at work are willing to listen to my personal problems. & \\
\hline
\end{tabular}

Table 7

Factor Loadings for Principal Components Factor Analysis with Oblimin Rotation of Anxiety

\begin{tabular}{lc}
\hline \multicolumn{1}{c}{ Item } & Anxiety \\
\hline My job gets to me more than it should. & .90 \\
Sometimes when I think about my job I get a tight feeling in my chest. & .88 \\
I have felt fidgety or nervous as a result of my job. & .88 \\
There are lots of times when my job drives me right up the wall. & .85 \\
\hline
\end{tabular}

Table 8

Factor Loadings for Principal Components Factor Analysis with Oblimin Rotation of Affective Commitment

\begin{tabular}{lc}
\hline \multicolumn{1}{c}{ Item } & $\begin{array}{c}\text { Affective } \\
\text { Commitment }\end{array}$ \\
\hline This organization has a great deal of personal meaning to me. & .83 \\
I would be very happy to spend the rest of my career with this & .81 \\
$\quad$ organization. & .79 \\
I do not feel "emotionally attached" to this organization. (R) & .73 \\
I do not feel a strong send of belonging to my organization. (R) & .59 \\
I enjoy discussing my organization with people outside of it. & .56 \\
I do not feel like "part of the family" at my organization. (R) & .49 \\
I think that I could easily become as attached to another organization as & .43 \\
$\quad$ I am to this one. (R) & \\
I really feel as if this organization's problems are my own. &
\end{tabular}


Table 9

Factor Loadings for Principal Components Factor Analysis with Oblimin Rotation of Intention to Leave

\begin{tabular}{lc}
\hline Item & Intention to Leave \\
\hline I will probably look for a new job in the next year. & .93 \\
I will actively look for a new job the next year. & .90 \\
I often think about quitting. & .83 \\
\hline
\end{tabular}

Table 10

Factor Loadings for Principal Components Factor Analysis with Oblimin Rotation of General Health

\begin{tabular}{lc}
\hline Item & General Health \\
\hline I have been feeling unhappy or depressed. (R) & .80 \\
I have been losing confidence in myself. (R) & .76 \\
I have been thinking of myself as a worthless person. (R) & .72 \\
I have felt that I am playing a useful part in things. & .66 \\
I have been able to face up to my problems. & .63 \\
I have felt capable of making decisions about things. & .63 \\
I have lost much sleep over worry. (R) & .63 \\
I have been feeling reasonably happy, all things & .61 \\
$\quad$ considered. & .58 \\
I have been able to enjoy my normal day-to-day activities. & .54 \\
I have felt I can't overcome my difficulties. (R) & .32 \\
I have been able to concentrate on what I am doing. &
\end{tabular}


Table 11

Factor Loadings for Principal Components Factor Analysis with Oblimin Rotation of Burnout

\begin{tabular}{lc}
\hline \multicolumn{1}{c}{ Item } & Burnout \\
\hline Helpless & .80 \\
Hopeless & .79 \\
Trapped & .78 \\
Depressed & .77 \\
Worthless / Like a failure & .77 \\
"I've had it" & .71 \\
Physically weak / Sickly & .65 \\
Difficulties sleeping & .59 \\
Tired & .59 \\
Disappointed with people & .53 \\
\hline
\end{tabular}

\section{Moderating Effects of Social Support}

Hypothesis $2\left(\mathrm{H}_{2}\right)$ postulated that the stressor-outcome relationship would be moderated by social support (coworker and supervisor), such that the effects of stressors on outcomes would be more deleterious in the presence of low social support, than in the presence of high social support. Preliminary analyses identified which variables ought to be used as controls for each dependent variable to test the hypotheses. It was determined that the variable important to control for in the regressions of anxiety was marital status $\left(F_{(4,130)}=2.85, p<.05\right)$, because married/re-married nurses indicated more anxiety than divorced/separated nurses. Age was controlled for in the regressions of affective commitment because as nurses' age increased their affective commitment increased as well $(r=.23, p<.05)$. Professional tenure also positively correlated with affective commitment $(r=.22, p<.05)$, such that affective commitment increased with the increase of the nurses' professional tenure. However, it was excluded as a control 
variable because of its high inter-correlation with age $(r=.79, p<.01)$, which could promote multicollinearity in later inferential analyses. Organizational tenure and job purpose were controlled for in regressions of intention to leave $\left(r=-.23, p<.05\right.$, and $F_{(1 \text {, }}$ 130) $=4.5, p<.05$, respectively), because as nurses' organizational tenure increased, their intention to leave decreased and nurses whose job purpose was direct patient care had lower intention to leave compared to nurses in non-direct patient care functions. Age and tenure in unit were excluded as controls despite their correlation with intention to leave, due to their high correlations with organizational tenure, to avoid multicollinearity effects. All remaining correlations between demographics and outcome variables were non-significant; none of the demographic variables analyzed as potential control variables showed a relationship with general health, job satisfaction or burnout. Table 12 summarizes the variables used as controls in subsequent inferential analyses.

Table 12

Summary of Control Variables Used in Inferential Analyses (Regressions) for each Outcome

\begin{tabular}{ll}
\hline \multicolumn{1}{c}{ Outcomes } & \multicolumn{1}{c}{ Control Variables } \\
\hline Anxiety & Marital Status \\
Affective Commitment & Age \\
Intention to Leave & Org. Tenure + Job Purpose \\
General Health & N/A \\
Job Satisfaction & N/A \\
Burnout & N/A \\
\hline Note. Marital Status is represented by four dummy variables (Single, Married/Re- \\
married, Separated/Divorced, and Widowed/Widower; additional option was Living with \\
Partner). Job purpose refers to whether or not the nurse provided direct care to patients.
\end{tabular}


A moderated multiple regression analysis was conducted to test Hypothesis 2 that social support (either coworker or supervisor support) would moderate the stressoroutcome relationship (see Table 13 through Table 18). A hierarchical (sequential) multiple regression analysis was performed for each of the dependent variables, with the control variables in step one (when applicable), the three role stressors in step two, supervisor and coworker social support variables in step three, and variables representing interactions between stressors and support variables in step four. The reason for including the stressor/support interaction variables in the last step derived from the hypothesis being tested, namely that the interaction of social support with stressors would account for more variance in outcomes/strains above and beyond the variance accounted for by the main effects of social support and role stressors.

Each of six outcome variables was regressed on role stressors and each social support variable (supervisor and coworker). For all six outcomes, the results showed a non-significant change in variance when the interaction terms of each role stressor and each social support variable were introduced in the last step of the regression. In other words, the stressors-social support interactions did not contribute significantly to explaining the variance in any of the six outcome study variables, above and beyond the explanatory power of the social support and stressor main effects. Hypothesis 2, therefore, was not supported. 
Table 13

Anxiety Regressed on Role Stressors, Social Supports, and their Interaction

\begin{tabular}{llll}
\hline \multicolumn{1}{c}{ Predictor } & $R^{2}$ & $\Delta R^{2}$ & $\beta$ \\
\hline Step 1 Control Variables ${ }^{1}$ & $.08^{*}$ & $.08^{*}$ & \\
Step 2 & $.30^{* * *}$ & $.22^{* * *}$ & \\
$\quad$ Role Overload (RO) & & & $.27^{* *}$ \\
Role Conflict (RC) & & $.23^{* *}$ \\
Role Ambiguity (RA) & & & .11 \\
Step 3 & $.31^{* * *}$ & .01 & \\
Supervisor Support (SupSup) & & & .07 \\
Coworker Support (CoSup) & & & -.08 \\
Step 4 & $.35^{* * *}$ & .04 & \\
RO $\times$ SupSup & & & .09 \\
RO $\times$ CoSup & & .31 \\
RC $\times$ SupSup & & .37 \\
RC $\times$ CoSup & & .67 \\
RA $\times$ SupSup & & .02 \\
RA $\times$ CoSup & & & .09 \\
\hline
\end{tabular}

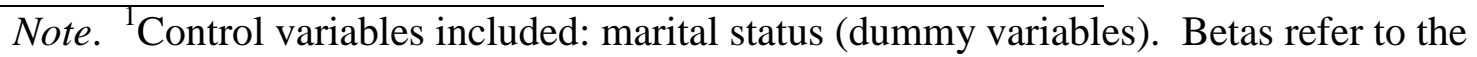
step at which the predictor was first entered.

${ }^{\mathrm{T}} p<.10 . *{ }^{*} p<.05$. *** $p<.01$. *** $p<.001$. 
Table 14

Affective Commitment Regressed on Role Stressors, Social Supports, and their Interaction

\begin{tabular}{|c|c|c|c|}
\hline Predictor & $R^{2}$ & $\Delta R^{2}$ & $\beta$ \\
\hline Step 1 Control Variable ${ }^{1}$ & $.05^{*}$ & $.05^{*}$ & $.22 *$ \\
\hline Step 2 & $.22 * * *$ & $.17 * * *$ & \\
\hline Role Overload (RO) & & & $-.17^{\mathrm{T}}$ \\
\hline Role Conflict (RC) & & & $-.16^{\mathrm{T}}$ \\
\hline Role Ambiguity (RA) & & & $-.21 *$ \\
\hline Step 3 & $.32 * * *$ & $.10 * * *$ & \\
\hline Supervisor Support (SupSup) & & & $.25 * *$ \\
\hline Coworker Support (CoSup) & & & $.24 * *$ \\
\hline Step 4 & $.34 * * *$ & .02 & \\
\hline RO $\times$ SupSup & & & -.06 \\
\hline $\mathrm{RO} \times \mathrm{CoSup}$ & & & .20 \\
\hline $\mathrm{RC} \times$ SupSup & & & .23 \\
\hline $\mathrm{RC} \times \mathrm{CoSup}$ & & & -.56 \\
\hline RA $\times$ SupSup & & & .23 \\
\hline $\mathrm{RA} \times \mathrm{CoSup}$ & & & -.22 \\
\hline
\end{tabular}


Table 15

Intention to Leave Regressed on Role Stressors, Social Supports, and their Interaction

\begin{tabular}{llll}
\hline \multicolumn{1}{c}{ Predictor } & $R^{2}$ & $\Delta R^{2}$ & $\beta$ \\
\hline Step 1 Control Variables ${ }^{*}$ & $.08^{* *}$ & $.08^{* *}$ & \\
Step 2 & $.24^{* * *}$ & $.17^{* * *}$ & \\
Role Overload (RO) & & & $.24^{* *}$ \\
Role Conflict (RC) & & .04 \\
Role Ambiguity (RA) & & & $.25^{* *}$ \\
Step 3 & $.25^{* * *}$ & .00 & \\
Supervisor Support (SupSup) & & & -.06 \\
Coworker Support (CoSup) & & & -.02 \\
Step 4 & $.28^{* * *}$ & .03 & \\
RO $\times$ SupSup & & & -.43 \\
RO $\times$ CoSup & & .44 \\
RC $\times$ SupSup & & .20 \\
RC $\times$ CoSup & & -.03 \\
RA $\times$ SupSup & & -.16 \\
RA $\times$ CoSup & & & -.34 \\
\hline
\end{tabular}

Note. ${ }^{1}$ Control variables included: organizational tenure and job purpose. Betas refer to the step at which the predictor was first entered.

${ }^{\mathrm{T}} p<.10 . *{ }^{*} p<.05$. *** $p<.01$. *** $p<.001$. 
Table 16

General Health Regressed on Role Stressors, Social Supports, and their Interaction

\begin{tabular}{llll}
\hline \multicolumn{1}{c}{ Predictor } & $R^{2}$ & $\Delta R^{2}$ & $\beta$ \\
\hline Step 1 & $.20^{* * *}$ & $.18^{* * *}$ & \\
Role Overload (RO) & & & -.10 \\
Role Conflict (RC) & & -.13 \\
Role Ambiguity (RA) & & & $-.30^{* *}$ \\
Step 2 & $.23^{* * *}$ & $.06^{* *}$ & \\
Supervisor Support (SupSup) & & & $-.19^{*}$ \\
Coworker Support (CoSup) & & & $.21^{*}$ \\
Step 3 & $.26^{* * * *}$ & .02 & \\
RO $\times$ SupSup & & & .38 \\
RO $\times$ CoSup & & -.10 \\
RC $\times$ SupSup & & -.37 \\
RC $\times$ CoSup & & -.52 \\
RA $\times$ SupSup & & -.06 \\
RA $\times$ CoSup & & .25 \\
\hline
\end{tabular}

Note. Betas refer to the step at which the predictor was first entered.

${ }^{\mp} p<.10 . * p<.05$. *** $p<.01$. *** $p<.001$. 
Table 17

Job Satisfaction Regressed on Role Stressors, Social Supports, and their Interaction

\begin{tabular}{|c|c|c|c|}
\hline Predictor & $R^{2}$ & $\Delta R^{2}$ & $\beta$ \\
\hline Step 1 & $.26 * * *$ & $.26 * * *$ & \\
\hline Role Overload (RO) & & & $-.28 * *$ \\
\hline Role Conflict (RC) & & & -.04 \\
\hline Role Ambiguity (RA) & & & $-.32 * * *$ \\
\hline Step 2 & $.27 * * *$ & .01 & \\
\hline Supervisor Support (SupSup) & & & .13 \\
\hline Coworker Support (CoSup) & & & .01 \\
\hline Step 3 & $.28 * * *$ & .01 & \\
\hline RO $\times$ SupSup & & & .22 \\
\hline $\mathrm{RO} \times \mathrm{CoSup}$ & & & -.28 \\
\hline $\mathrm{RC} \times$ SupSup & & & .04 \\
\hline $\mathrm{RC} \times \mathrm{CoSup}$ & & & -.05 \\
\hline RA $\times$ SupSup & & & .12 \\
\hline $\mathrm{RA} \times \mathrm{CoSup}$ & & & -.08 \\
\hline
\end{tabular}

Note. Betas refer to the step at which the predictor was first entered.

${ }^{\mp} p<.10 . * p<.05$. *** $p<.01$. *** $p<.001$. 
Table 18

Burnout Regressed on Role Stressors, Social Supports, and their Interaction

\begin{tabular}{lccc}
\hline \multicolumn{1}{c}{ Predictor } & $R^{2}$ & $\Delta R^{2}$ & $\beta$ \\
\hline Step 1 & $.15^{* * *}$ & $.15^{* * *}$ & \\
Role Overload (RO) & & & $.24^{* *}$ \\
Role Conflict (RC) & & & $.15^{\text {中 }}$ \\
Role Ambiguity (RA) & & & .11 \\
Step 2 & $.18^{* * *}$ & $.03^{\dagger}$ & \\
Supervisor Support (SupSup) & & & .09 \\
Coworker Support (CoSup) & & & $-.17^{*}$ \\
Step 3 & $.20^{* *}$ & .02 & \\
RO $\times$ SupSup & & & -.42 \\
RO $\times$ CoSup & & & .60 \\
RC $\times$ SupSup & & & -.07 \\
RC $\times$ CoSup & & & -.12 \\
RA $\times$ SupSup & & & .08 \\
RA $\times$ CoSup & & & -.16 \\
\hline
\end{tabular}

Note. Betas refer to the step at which the predictor was first entered.

${ }^{\mp} p<.10 . * p<.05$. ***p $<.01$. *** $p<.001$.

\section{Moderating Effects of Social Support in Interaction with Self-Construal}

Hypothesis $3\left(H_{3}\right)$ proposed there would be a three-way interaction of coworker social support, role stressors, and self-construal in predicting outcomes, such that for individuals high on interdependent self-construal the relationship between role stressors and outcomes would be weaker for individuals with high coworker support than with low coworker support. Hypothesis $4\left(H_{4}\right)$ postulated there would be a three-way interaction of supervisor social support, role stressors, and self-construal in predicting outcomes, such that for individuals high on independent self-construal the relationship between role 
stressors and outcomes would be weaker for individuals with high supervisor social support than with low supervisor social support.

A moderated multiple regression analysis was employed to test $H_{3}$ and $H_{4}$. A preliminary moderated hierarchical (sequential) multiple regression analysis was performed for each of the dependent variables, with the control variables in step one (when applicable), the three role stressors in step two, supervisor support, coworker support, and self-construal variables in step three, the variables representing the two-way interaction between stressors, support, and self-construal in step four, and the variables representing the three-way interaction between stressors, support and self-construal in step five. The reason for including the stressor/support/self-construal interaction variables in the last step derived from the hypotheses being tested, namely that social support (resource), stressors (situation) and self-construal (context) would interact, to determine if adding context to the interaction of social support and stressors would better explain the context in which social support would moderate the stressor-strain relationship. Outcome variables that were explained by the three-way interaction were subjected to further analyses to understand the direction of the interaction.

Tables 19 through 24 depict the results of the moderated regressions of each of the six outcomes (dependent variables) on the three role stressors, social support (supervisor and coworker), and self-construal. Results showed an overall significant relationship between all the predictors and the outcomes ( $R^{2}$ at the last step). However, only for anxiety (Table 19), general health (Table 22), and burnout (Table 24) was there a significant three-way interaction term in the final step. For anxiety, it was the interaction 
between role overload, self-construal, and coworker support (RO x SC x CoSup) that showed a statistically significant unique contribution of variance (above and beyond other tested relationships; $\beta=-4.10, p<.05)$. The interaction of role ambiguity, selfconstrual, and supervisor support (RA x SC x SupSup) showed a significant unique contribution of variance in general health $(\beta=-2.35, p<.05)$, as well as in Burnout $(\beta=$ $3.15, p<.01)$.

Significant three-way interactions were further tested to understand the nature and direction of these identified relationships. A moderated hierarchical multiple regression analysis was performed for anxiety, general health, and burnout, with the control variables in step one (when applicable), the role stressor involved in the significant interaction in step two, self-construal and the type of social support involved in the significant interaction in step three, the variables representing the two-way interactions between stressor, support, and self-construal in step four, and the variable representing the previously significant three-way interaction in step five. 
Table 19

Anxiety Regressed on Role Stressors, Social Supports, Self-Construal, and their Interaction

\begin{tabular}{|c|c|c|c|}
\hline Predictor & $R^{2}$ & $\Delta R^{2}$ & $\beta$ \\
\hline Step 1 Control variables ${ }^{1}$ & $.08^{*}$ & $.08 *$ & \\
\hline Step 2 & $.30 * * *$ & $.22 * * *$ & \\
\hline Role Overload (RO) & & & $.27 * *$ \\
\hline Role Conflict (RC) & & & $.23 * *$ \\
\hline Role Ambiguity (RA) & & & .11 \\
\hline Step 3 & $.33 * * *$ & .03 & \\
\hline Supervisor Support (SupSup) & & & .05 \\
\hline Coworker Support (CoSup) & & & -.09 \\
\hline Self-Construal (SC) & & & -.15 \\
\hline Step 4 & $.39 * * *$ & .06 & \\
\hline $\mathrm{RO} \times \mathrm{SC}$ & & & .16 \\
\hline $\mathrm{RO} \times$ SupSup & & & .03 \\
\hline $\mathrm{RO} \times \mathrm{CoSup}$ & & & .28 \\
\hline $\mathrm{RC} \times \mathrm{SC}$ & & & $-.85^{\mathrm{T}}$ \\
\hline $\mathrm{RC} \times$ SupSup & & & .23 \\
\hline $\mathrm{RC} \times \mathrm{CoSup}$ & & & .70 \\
\hline $\mathrm{RA} \times \mathrm{SC}$ & & & .28 \\
\hline $\mathrm{RA} \times$ SupSup & & & .01 \\
\hline $\mathrm{RA} \times \mathrm{CoSup}$ & & & .15 \\
\hline $\mathrm{SC} \times$ SupSup & & & -.48 \\
\hline $\mathrm{SC} \times \mathrm{CoSup}$ & & & -.26 \\
\hline Step 5 & $.45 * * *$ & $.07^{\mathrm{T}}$ & \\
\hline $\mathrm{RO} \times \mathrm{SC} \times \mathrm{SupSup}$ & & & -.55 \\
\hline $\mathrm{RO} \times \mathrm{SC} \times \mathrm{CoSup}$ & & & $-4.10 *$ \\
\hline $\mathrm{RC} \times \mathrm{SC} \times \mathrm{SupSup}$ & & & -1.26 \\
\hline $\mathrm{RC} \times \mathrm{SC} \times \mathrm{CoSup}$ & & & 3.30 \\
\hline $\mathrm{RA} \times \mathrm{SC} \times \mathrm{SupSup}$ & & & 1.61 \\
\hline $\mathrm{RA} \times \mathrm{SC} \times \mathrm{CoSup}$ & & & -2.67 \\
\hline
\end{tabular}

Note. ${ }^{1}$ Control variables included: marital status (dummy variables). Betas refer to the step at which the predictor was first entered.

${ }^{\mathrm{T}} p<.10 .{ }^{*} p<.05$. ** $p<.01$. ***p $<.001$. 
Table 20

Affective Commitment Regressed on Role Stressors, Social Supports, Self-Construal, and their Interaction

\begin{tabular}{|c|c|c|c|}
\hline Predictor & $R^{2}$ & $\Delta R^{2}$ & $\beta$ \\
\hline Step 1 Control variable ${ }^{1}$ & $.05^{*}$ & $.05^{*}$ & $.22 *$ \\
\hline Step 2 & $.22 * * *$ & $.17 * * *$ & \\
\hline Role Overload (RO) & & & $-.17^{\mathrm{t}}$ \\
\hline Role Conflict (RC) & & & $-.16^{\mathrm{t}}$ \\
\hline Role Ambiguity (RA) & & & $-.21 *$ \\
\hline Step 3 & $.32 * * *$ & $.10^{* * *}$ & \\
\hline Supervisor Support (SupSup) & & & $.26 * *$ \\
\hline Coworker Support (CoSup) & & & $.24 * *$ \\
\hline Self-Construal (SC) & & & .04 \\
\hline Step 4 & $.37 * * *$ & .05 & \\
\hline $\mathrm{RO} \times \mathrm{SC}$ & & & .02 \\
\hline $\mathrm{RO} \times$ SupSup & & & .08 \\
\hline $\mathrm{RO} \times \mathrm{CoSup}$ & & & .05 \\
\hline $\mathrm{RC} \times \mathrm{SC}$ & & & -.74 \\
\hline $\mathrm{RC} \times$ SupSup & & & .05 \\
\hline $\mathrm{RC} \times \mathrm{CoSup}$ & & & -.39 \\
\hline $\mathrm{RA} \times \mathrm{SC}$ & & & .22 \\
\hline RA $\times$ SupSup & & & .27 \\
\hline $\mathrm{RA} \times \mathrm{CoSup}$ & & & -.12 \\
\hline SC $\times$ SupSup & & & .11 \\
\hline $\mathrm{SC} \times \mathrm{CoSup}$ & & & .18 \\
\hline Step 5 & $.38^{* *}$ & .01 & \\
\hline $\mathrm{RO} \times \mathrm{SC} \times \mathrm{SupSup}$ & & & -1.02 \\
\hline $\mathrm{RO} \times \mathrm{SC} \times \mathrm{CoSup}$ & & & .06 \\
\hline $\mathrm{RC} \times \mathrm{SC} \times$ SupSup & & & .04 \\
\hline $\mathrm{RC} \times \mathrm{SC} \times \mathrm{CoSup}$ & & & .68 \\
\hline $\mathrm{RA} \times \mathrm{SC} \times$ SupSup & & & .27 \\
\hline $\mathrm{RA} \times \mathrm{SC} \times \mathrm{CoSup}$ & & & -1.56 \\
\hline
\end{tabular}

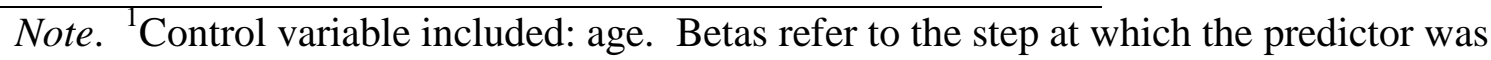
first entered.

${ }^{\mp} p<.10 . * p<.05 . * * p<.01 . * * * p<.001$. 
Table 21

Intention to Leave Regressed on Role Stressors, Social Supports, Self-Construal, and their Interaction

\begin{tabular}{|c|c|c|c|}
\hline Predictor & $R^{2}$ & $\Delta R^{2}$ & $\beta$ \\
\hline Step 1 Control variables ${ }^{\top}$ & $.08 * *$ & $.08 * *$ & \\
\hline Step 2 & $.24 * * *$ & $.17 * * *$ & \\
\hline Role Overload (RO) & & & $.24 * *$ \\
\hline Role Conflict (RC) & & & .04 \\
\hline Role Ambiguity (RA) & & & $.25 * *$ \\
\hline Step 3 & $.25 * * *$ & .01 & \\
\hline Supervisor Support (SupSup) & & & -.06 \\
\hline Coworker Support (CoSup) & & & -.03 \\
\hline Self-Construal (SC) & & & -.08 \\
\hline Step 4 & $.31 * *$ & .06 & \\
\hline $\mathrm{RO} \times \mathrm{SC}$ & & & .13 \\
\hline $\mathrm{RO} \times \mathrm{SupSup}$ & & & -.55 \\
\hline $\mathrm{RO} \times$ CoSup & & & .49 \\
\hline $\mathrm{RC} \times \mathrm{SC}$ & & & -.21 \\
\hline $\mathrm{RC} \times$ SupSup & & & .19 \\
\hline $\mathrm{RC} \times \mathrm{CoSup}$ & & & -.09 \\
\hline $\mathrm{RA} \times \mathrm{SC}$ & & & -.03 \\
\hline RA $\times$ SupSup & & & -.18 \\
\hline $\mathrm{RA} \times \mathrm{CoSup}$ & & & -.40 \\
\hline SC $\times$ SupSup & & & -.45 \\
\hline $\mathrm{SC} \times \mathrm{CoSup}$ & & & -.45 \\
\hline Step 5 & $.34 * *$ & .03 & \\
\hline $\mathrm{RO} \times \mathrm{SC} \times \mathrm{SupSup}$ & & & .64 \\
\hline $\mathrm{RO} \times \mathrm{SC} \times \mathrm{CoSup}$ & & & -2.09 \\
\hline $\mathrm{RC} \times \mathrm{SC} \times \mathrm{SupSup}$ & & & 1.84 \\
\hline $\mathrm{RC} \times \mathrm{SC} \times \mathrm{CoSup}$ & & & 3.32 \\
\hline $\mathrm{RA} \times \mathrm{SC} \times \mathrm{SupSup}$ & & & .06 \\
\hline $\mathrm{RA} \times \mathrm{SC} \times \mathrm{CoSup}$ & & & -1.24 \\
\hline
\end{tabular}

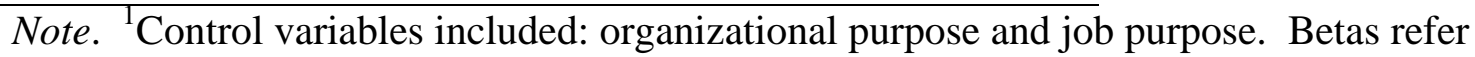
to the step at which the predictor was first entered.

${ }^{\mathrm{T}} p<.10 . * p<.05$. ** $p<.01$. ***p $<.001$. 
Table 22

General Health Regressed on Role Stressors, Social Supports, Self-Construal, and their Interaction

\begin{tabular}{|c|c|c|c|}
\hline Predictor & $R^{2}$ & $\Delta R^{2}$ & $\beta$ \\
\hline Step 1 & $.18 * * *$ & $.18 * * *$ & \\
\hline Role Overload (RO) & & & -.10 \\
\hline Role Conflict (RC) & & & -.13 \\
\hline Role Ambiguity (RA) & & & $-.30 * *$ \\
\hline Step 2 & $.24 * * *$ & $.06^{*}$ & \\
\hline Supervisor Support (SupSup) & & & $-.18 *$ \\
\hline Coworker Support (CoSup) & & & $.22 * *$ \\
\hline Self-Construal (SC) & & & .07 \\
\hline Step 3 & $.30 * * *$ & .06 & \\
\hline $\mathrm{RO} \times \mathrm{SC}$ & & & -.61 \\
\hline RO $\times$ SupSup & & & .36 \\
\hline $\mathrm{RO} \times \mathrm{CoSup}$ & & & -.14 \\
\hline $\mathrm{RC} \times \mathrm{SC}$ & & & .27 \\
\hline $\mathrm{RC} \times$ SupSup & & & -.24 \\
\hline $\mathrm{RC} \times \mathrm{CoSup}$ & & & -.64 \\
\hline $\mathrm{RA} \times \mathrm{SC}$ & & & .12 \\
\hline $\mathrm{RA} \times$ SupSup & & & -.01 \\
\hline $\mathrm{RA} \times \mathrm{CoSup}$ & & & .35 \\
\hline SC $\times$ SupSup & & & .21 \\
\hline $\mathrm{SC} \times \mathrm{CoSup}$ & & & .61 \\
\hline Step 4 & $.34 * *$ & .05 & \\
\hline $\mathrm{RO} \times \mathrm{SC} \times \mathrm{SupSup}$ & & & .21 \\
\hline $\mathrm{RO} \times \mathrm{SC} \times \mathrm{CoSup}$ & & & 1.50 \\
\hline $\mathrm{RC} \times \mathrm{SC} \times \mathrm{SupSup}$ & & & 1.12 \\
\hline $\mathrm{RC} \times \mathrm{SC} \times \mathrm{CoSup}$ & & & -1.73 \\
\hline $\mathrm{RA} \times \mathrm{SC} \times$ SupSup & & & $-2.35 *$ \\
\hline $\mathrm{RA} \times \mathrm{SC} \times \mathrm{CoSup}$ & & & .03 \\
\hline
\end{tabular}

Note. Betas refer to the step at which the predictor was first entered. ${ }^{\mathrm{T}} p<.10 .{ }^{*} p<.05$. $* * p<.01 .{ }^{*} * * p<.001$. 
Table 23

Job Satisfaction Regressed on Role Stressors, Social Supports, Self-Construal, and their Interaction

\begin{tabular}{|c|c|c|c|}
\hline Predictor & $R^{2}$ & $\Delta R^{2}$ & $\beta$ \\
\hline Step 1 & $.26 * * *$ & $.26 * * *$ & \\
\hline Role Overload (RO) & & & $-.28 * *$ \\
\hline Role Conflict (RC) & & & -.04 \\
\hline Role Ambiguity (RA) & & & $-.32 * * *$ \\
\hline Step 2 & $.27 * * *$ & .01 & \\
\hline Supervisor Support (SupSup) & & & .13 \\
\hline Coworker Support (CoSup) & & & .01 \\
\hline Self-Construal (SC) & & & -.02 \\
\hline Step 3 & $.30 * * *$ & .03 & \\
\hline $\mathrm{RO} \times \mathrm{SC}$ & & & -.23 \\
\hline RO $\times$ SupSup & & & .18 \\
\hline $\mathrm{RO} \times \mathrm{CoSup}$ & & & -.40 \\
\hline $\mathrm{RC} \times \mathrm{SC}$ & & & .09 \\
\hline $\mathrm{RC} \times$ SupSup & & & .02 \\
\hline $\mathrm{RC} \times \mathrm{CoSup}$ & & & -.11 \\
\hline $\mathrm{RA} \times \mathrm{SC}$ & & & .47 \\
\hline $\mathrm{RA} \times$ SupSup & & & .19 \\
\hline $\mathrm{RA} \times \mathrm{CoSup}$ & & & -.11 \\
\hline $\mathrm{SC} \times$ SupSup & & & .56 \\
\hline $\mathrm{SC} \times \mathrm{CoSup}$ & & & -.45 \\
\hline Step 4 & $.32 * *$ & .02 & \\
\hline $\mathrm{RO} \times \mathrm{SC} \times \mathrm{SupSup}$ & & & -.32 \\
\hline $\mathrm{RO} \times \mathrm{SC} \times \mathrm{CoSup}$ & & & -.12 \\
\hline $\mathrm{RC} \times \mathrm{SC} \times \mathrm{SupSup}$ & & & -2.31 \\
\hline $\mathrm{RC} \times \mathrm{SC} \times \mathrm{CoSup}$ & & & -3.00 \\
\hline $\mathrm{RA} \times \mathrm{SC} \times \mathrm{SupSup}$ & & & 1.19 \\
\hline $\mathrm{RA} \times \mathrm{SC} \times \mathrm{CoSup}$ & & & .32 \\
\hline
\end{tabular}

Note. Betas refer to the step at which the predictor was first entered.

${ }^{\mathrm{T}} p<.10 .{ }^{*} p<.05$. ** $p<.01$. *** $p<.001$. 
Table 24

Burnout Regressed on Role Stressors, Social Supports, Self-Construal, and their Interaction

\begin{tabular}{|c|c|c|c|}
\hline Predictor & $R^{2}$ & $\Delta R^{2}$ & $\beta$ \\
\hline Step 1 & $.15 * * *$ & $.15 * * *$ & \\
\hline Role Overload (RO) & & & $.24 * *$ \\
\hline Role Conflict (RC) & & & $.15^{\mathrm{t}}$ \\
\hline Role Ambiguity (RA) & & & .11 \\
\hline Step 2 & $.18 * * *$ & .03 & \\
\hline Supervisor Support (SupSup) & & & .09 \\
\hline Coworker Support (CoSup) & & & $-.18 *$ \\
\hline Self-Construal (SC) & & & -.00 \\
\hline Step 3 & $.22 *$ & .03 & \\
\hline $\mathrm{RO} \times \mathrm{SC}$ & & & .36 \\
\hline RO $\times$ SupSup & & & -.33 \\
\hline $\mathrm{RO} \times \mathrm{CoSup}$ & & & .64 \\
\hline $\mathrm{RC} \times \mathrm{SC}$ & & & -.42 \\
\hline $\mathrm{RC} \times$ SupSup & & & -.17 \\
\hline $\mathrm{RC} \times \mathrm{CoSup}$ & & & -.00 \\
\hline $\mathrm{RA} \times \mathrm{SC}$ & & & -.27 \\
\hline RA $\times$ SupSup & & & .04 \\
\hline $\mathrm{RA} \times \mathrm{CoSup}$ & & & -.16 \\
\hline SC $\times$ SupSup & & & -.28 \\
\hline $\mathrm{SC} \times \mathrm{CoSup}$ & & & .04 \\
\hline Step 4 & $.30 * *$ & $.08 *$ & \\
\hline $\mathrm{RO} \times \mathrm{SC} \times \mathrm{SupSup}$ & & & -.21 \\
\hline $\mathrm{RO} \times \mathrm{SC} \times \mathrm{CoSup}$ & & & -.24 \\
\hline $\mathrm{RC} \times \mathrm{SC} \times \mathrm{SupSup}$ & & & -.31 \\
\hline $\mathrm{RC} \times \mathrm{SC} \times \mathrm{CoSup}$ & & & 2.23 \\
\hline $\mathrm{RA} \times \mathrm{SC} \times$ SupSup & & & $3.15 * *$ \\
\hline $\mathrm{RA} \times \mathrm{SC} \times \mathrm{CoSup}$ & & & -2.83 \\
\hline
\end{tabular}

Note. Betas refer to the step at which the predictor was first entered. ${ }^{\mathrm{T}} p<.10 .{ }^{*} p<.05$. $* * p<.01$. $* * * p<.001$. 
For anxiety, a moderated hierarchical multiple regression was conducted with marital status dummy variables in step one, role overload in step two, coworker support and self-construal in step three, the three two-way interactions between role overload, coworker support and self-construal in step four (RO x SC, RO x CoSup, and SC x CoSup) and, in step five, the three-way interaction variable (RO x SC x CoSup). Table 25 shows results from this moderated regression. The main effect for role overload was significant in Step 1, coworker support and self-construal did not add significant variance in Step 2, but the interaction of role overload and coworker support was significant, despite no added significant variance in anxiety and the variance explained by role overload having disappeared. In the final step, however, when all main effects and interaction effects were included, the overall variance explained in anxiety was significant $\left(R^{2}=.33, p<.001\right)$ and each of the variables were relevant to predicting anxiety. Further, change in variance in anxiety due to the addition of the three-way interaction term was significant $\left(\Delta R^{2}=.04, p<.05\right)$.

Figure 6 depicts the nature of this interaction. Its values were calculated per Aiken and West's (1991) recommended procedures, using one standard deviation below and one standard deviation above the variables' means (Da Silva, Hutcheson, \& Wahl, 2010; Da Silva \& Oldham, 2012; Dawson, 2014). As the figure shows, for participants tending toward an interdependent self-construal (upper graph), the correlation between role overload and anxiety was positive for those with high coworker support but slightly (weakly) negative when coworker support was low. These results indicate $H_{3}$ was not supported in that the interaction occurred in the opposite direction of what was 
anticipated. Nonetheless, these results evidenced a three-way interaction of RO x SC x CoSup on anxiety, indicating that self-construal might matter in moderating the impact of social support.

Table 25

Anxiety Regressed on Role Overload, Coworker Social Support, Self-Construal, and their Interaction

\begin{tabular}{lllc}
\hline \multicolumn{1}{c}{ Predictor } & $R^{2}$ & $\Delta R^{2}$ & $\beta$ \\
\hline Step 1 Control variables & $.08^{*}$ & $.08^{*}$ & \\
Step 2 & $.23^{* * *}$ & .15 & \\
$\quad$ Role Overload (RO) & & & $.39 * * *$ \\
$\quad$ Step 3 & $.26^{* * *}$ & .03 & \\
$\quad$ Coworker Support (CoSup) & & & -.12 \\
$\quad$ Self-Construal (SC) & & & -.13 \\
Step 4 & $.29 * * *$ & .04 & \\
$\quad$ RO $\times$ SC & & & .12 \\
RO $\times$ CoSup & & & $.77^{*}$ \\
SC $\times$ CoSup & & & -.41 \\
Step 5 & $.33^{* * *}$ & $.04 *$ & \\
$\quad$ RO $\times$ SC $\times$ CoSup & & & $-3.99 *$
\end{tabular}

Note. ${ }^{1}$ Control variables included: marital status (dummy variables). Betas refer to the step at which the predictor was first entered.

${ }^{\mathrm{T}} p<.10 . * p<.05$. ** ${ }^{*} p<.01$. ***p $<.001$. 


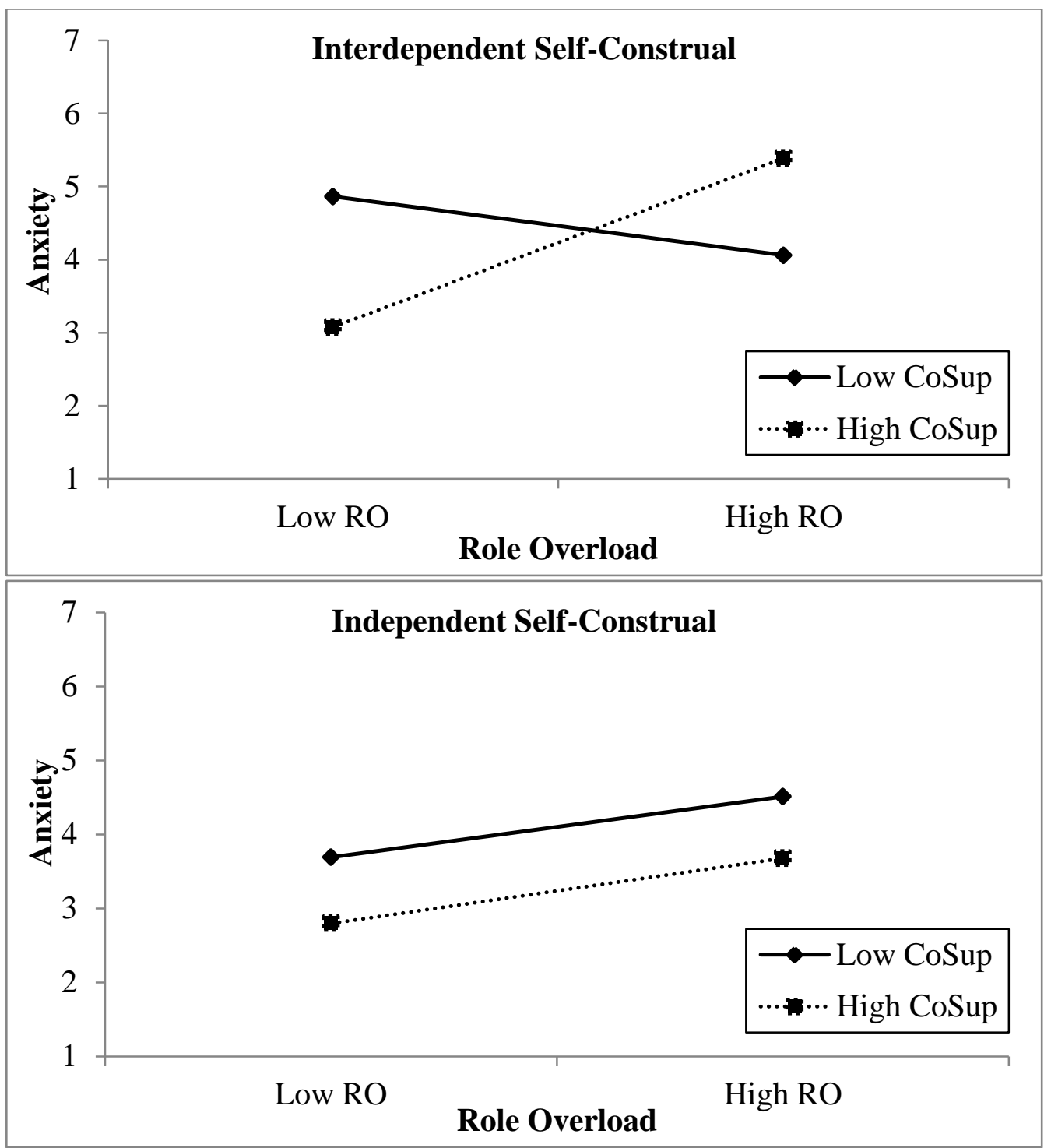

Figure 6. Interaction between Role Overload, Coworker Social Support, and SelfConstrual on Anxiety. Upper graphic depicts nature of significant interaction resulting from testing $\mathrm{H}_{3}$. 
Next, general health was regressed on role ambiguity (step one), supervisor support and self-construal (step two), the three two-way interactions between role ambiguity, supervisor support and self-construal (RA x SC, RA x SupSup, SC x SupSup; step three), and the three-way interaction (RA x SC x SupSup; step four). Per Table 26, supervisor support and self-construal did not add significant variance in Step 2 and the two-way interactions did not add significant variance in Step 3, above that which was already accounted for by role ambiguity. However, the three-way interaction did account for a significant change in variance $\left(\Delta R^{2}=.04, p<.05\right)$ in general health. Furthermore, when all main-effect and interaction-effect predictors were included in the final step, the overall variance explained in general health was significant $\left(R^{2}=.21, p<.001\right)$ and all the contributing variables had significant beta weights.

Figure 7 represents the nature of the interaction between role ambiguity, supervisor support and self-construal in predicting general health, and was designed using the same guidelines mentioned previously for the study of three-way interactions. For participants tending toward an independent self-construal (lower graph), there was a stronger negative relationship between role ambiguity and general health for those with high (vs. low) supervisor support. Therefore $H_{4}$ was not supported for general health in that the interaction occurred in the opposite direction of what was anticipated. Nonetheless, self-construal played a role in the extent to which social support moderated the role ambiguity-general health relationship. 
Table 26

General Health Regressed on Role Ambiguity, Supervisor Social Support, Self-Construal, and their Interaction

\begin{tabular}{lclc}
\hline \multicolumn{1}{c}{ Predictor } & $R^{2}$ & $\Delta R^{2}$ & $\beta$ \\
\hline Step 1 & $.14^{* * *}$ & $.14^{* * *}$ & \\
$\quad$ Role Ambiguity (RA) & & & $-.38^{* * *}$ \\
Step 2 & $.16^{* * * *}$ & .01 & \\
$\quad$ Supervisor Support (SupSup) & & & -.10 \\
$\quad$ Self-Construal (SC) & & & .07 \\
Step 3 & $.17^{* *}$ & .01 & \\
RA $\times$ SC & & & -.08 \\
RA $\times$ SupSup & & & -.06 \\
SC $\times$ SupSup & & & .32 \\
Step 4 & $.21 * * *$ & $.04 *$ & \\
RA $\times$ SC $\times$ SupSup & & & $-1.97 *$ \\
\hline
\end{tabular}

Note. Betas refer to the step at which the predictor was first entered.

${ }^{\mathrm{T}} p<.10 . * p<.05$. *** $p<.01$. *** $p<.001$. 


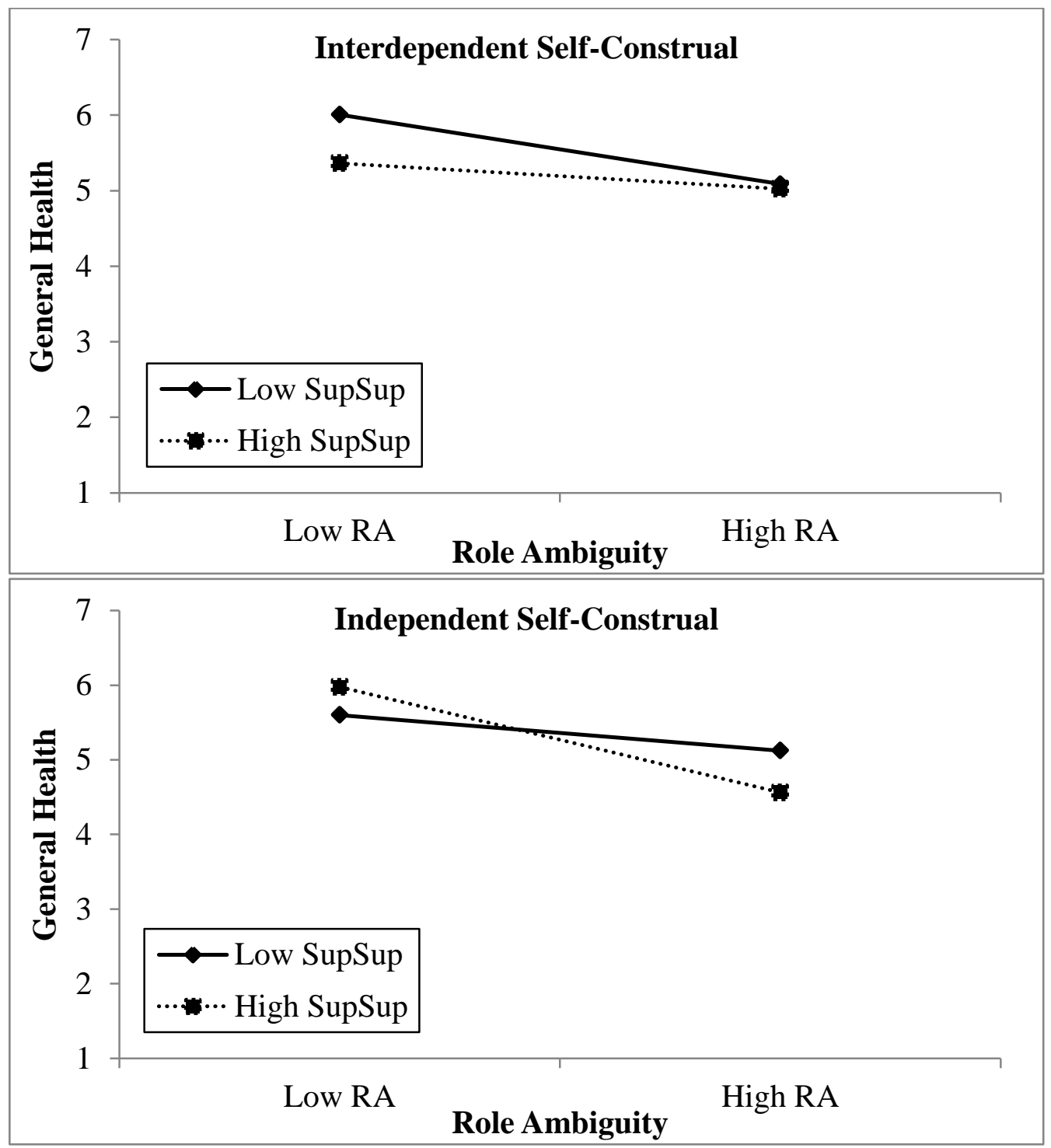

Figure 7. Interaction between Role Ambiguity, Supervisor Social Support, and SelfConstrual on General Health. Bottom graphic depicts nature of significant interaction resulting from testing $H_{4}$. 
As with general health, a moderated hierarchical multiple regression was conducted to predict the extent to which burnout could be explained by role ambiguity (RA), supervisor support (SupSup), and self-construal (SC), their two-way interactions (RA x SC, RA x SupSup, SC x SupSup) and, their three-way interaction (RA x SC x SupSup). Table 27 shows that, in Step 1, role ambiguity directly accounted for significant variance in burnout; however, supervisor support and self-construal did not add significant variance to that effect and, in Step 3, the two-way interactions also did not explain significant variance in burnout. Moreover, in Step 3, the beta weight for role ambiguity became non-significant. Nevertheless, in the final step, the overall relationship between all main effect and interaction effect predictors together explained a significant amount of variance in burnout $\left(R^{2}=.14, p<.01\right)$, and each of the beta weights, as well as, the addition of the three-way interaction was significant $\left(\Delta R^{2}=.07, p<.01\right)$.

Figure 8 represents the nature of the interactions, with values calculated per the recommended procedures. For participants tending toward an independent self-construal (lower graph), there was a stronger positive relationship between role ambiguity and burnout for those with high supervisor support than for those with low supervisor support. Therefore, $H_{4}$ was not supported for burnout, because the interaction occurred in the opposite direction of what was anticipated. However, that the three-way interaction between supervisor support, role ambiguity, and self-construal explained significant variance in burnout and caused each of the other terms in the regression to be significant, indicates that self-construal might play an important role in explaining when social support moderates the stressor-strain relationship. 
Table 27

Burnout Regressed on Role Ambiguity, Supervisor Social Support, Self-Construal, and their Interaction

\begin{tabular}{llll}
\hline \multicolumn{1}{c}{ Predictor } & $R^{2}$ & $\Delta R^{2}$ & $\beta$ \\
\hline Step 1 & $.06^{* *}$ & $.06^{* *}$ & \\
$\quad$ Role Ambiguity (RA) & & & $.25^{* *}$ \\
Step 2 & $.06^{* *}$ & .00 & \\
$\quad$ Supervisor Support (SupSup) & & & -.01 \\
$\quad$ Self-Construal (SC) & & & -.03 \\
Step 3 & .07 & .01 & \\
RA $\times$ SC & & & -.25 \\
RA $\times$ SupSup & & & -.01 \\
$\quad$ SC $\times$ SupSup & & & -.36 \\
Step 4 & $.14^{* *}$ & $.07 * *$ & \\
$\quad$ RA $\times$ SC $\times$ SupSup & & & $2.65^{* *}$ \\
\hline
\end{tabular}

Note. Betas refer to the step at which the predictor was first entered.

${ }^{\mathrm{T}} p<.10 . * p<.05$. *** $p<.01$. *** $p<.001$.

To summarize the study results, $H_{1}$, examining correlations between social support and study outcomes, was mostly supported, but $H_{2}$, focused on the moderating effect of social support on stressor-outcome relationships, was not supported.

Furthermore, $H_{3}$ and $H_{4}$, testing a three-way interaction between self-construal, social support, and stressors, were also not supported in the hypothesized direction. However, some of the three-way interactions explained significant variance in anxiety, general health, and burnout, just not in the expected direction. 


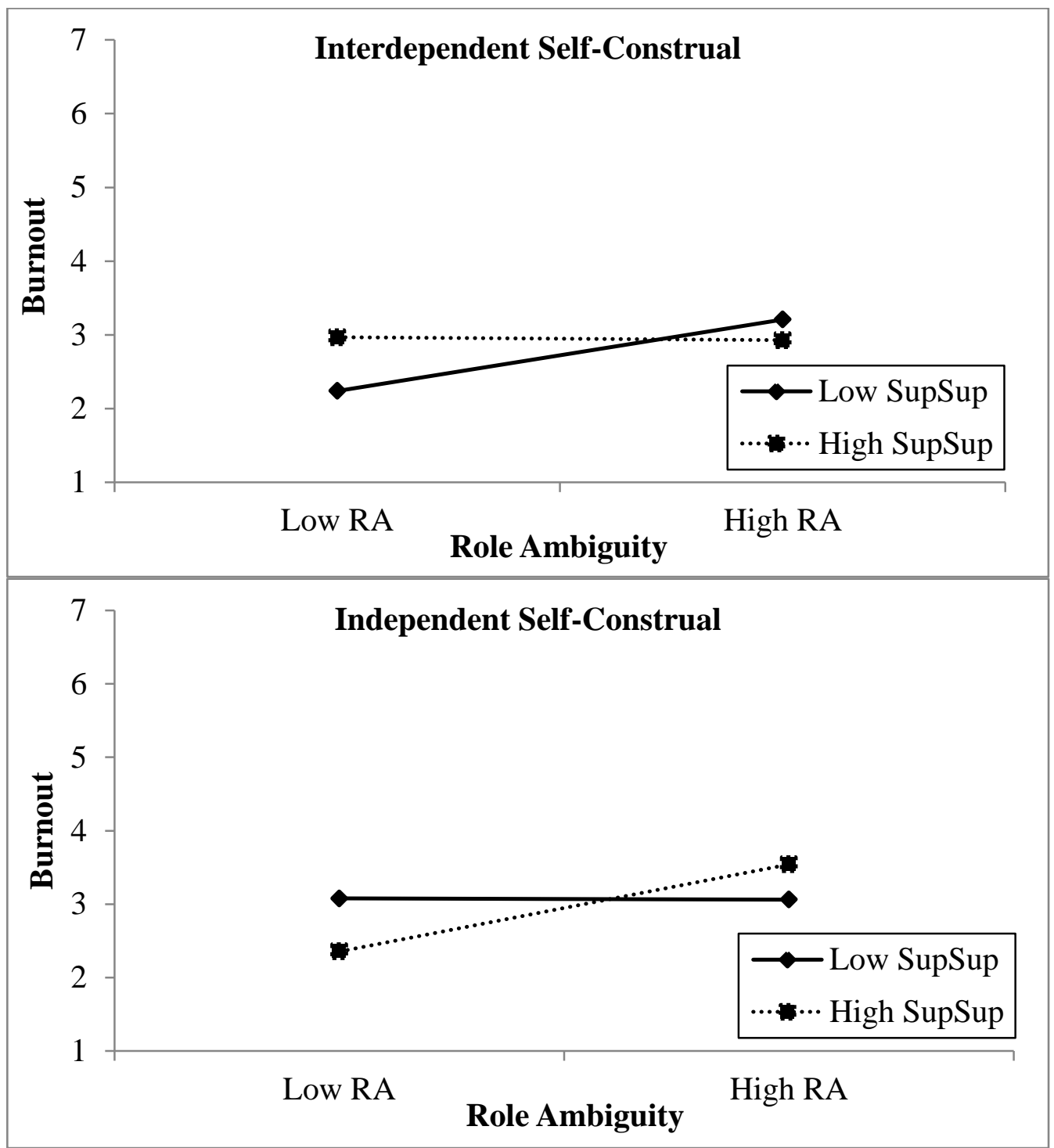

Figure 8. Interaction between Role Ambiguity, Supervisor Social Support, and SelfConstrual on Burnout. Bottom graphic depicts nature of significant interaction resulting from testing $\mathrm{H}_{4}$. 


\section{Discussion}

The purpose of this study was to test the extent to which self-construal might explain when social support moderates relationships between stressors at Time 1 and outcomes at Time 2. Prior literature and research have provided inconsistent, and sometimes contradictory, results on the direction in which social support moderates stressor-strain relationships, that is, whether it buffers or reverse buffers the relationship. Additionally, literature has seldom investigated the moderating role of social support on a longitudinal dataset with stressors collected at Time 1 and outcomes at Time 2 (exceptions include Dormann \& Zapf, 1999, and Nahum-Shani \& Bamberger, 2011). It is particularly important to study further the role of social support because it is often prescribed in interventions as a panacea to occupational stress. Although few researchers have engaged in identifying the context in which social support plays a protective role (e.g., Beehr et al., 1990, studied the content of support providers' communication), the present study begins to peel the layers of context from the lens of the person who receives (or does not receive) the support, by studying how individuals perceive themselves in relation to others. In other words, this study investigates the context in which social support mitigates, aggravates, or adds no effects on stressor-outcome relationships.

Glazer's (2006) work began to show that people in individualistic and collectivistic cultures perceived supervisory and coworker support differently. Extending from her study, this thesis proposed that interdependent and independent self-construals (Markus \& Kitayama, 1991), as internalized representations of culture (collectivism and individualism, respectively; Markus \& Kitayama, 1991; 2010; Oyserman, 2011), would 
explain when supervisor and coworker social support moderate the relationship between stressors (role overload, role ambiguity, and role conflict) and outcomes (anxiety, affective commitment, intention to leave, general health, job satisfaction, and burnout).

Before testing these three-way interactions, it was important to start by testing direct effects $\left(H_{1}\right)$ and simple moderator effect $\left(H_{2}\right)$ hypotheses. To replicate the common literature findings that social support has a direct beneficial impact on outcomes, $H_{1}$ was first tested. $H_{1}$ purported that supervisor and coworker social support was associated with lower levels of unfavorable outcomes (anxiety, intention to leave, and burnout) and with higher levels of favorable outcomes (affective commitment, general health, and job satisfaction). This hypothesis was supported such that when the surveyed nurses reported higher levels of coworker social support they also reported higher levels of affective commitment, general health, and job satisfaction, and lower levels of anxiety, and burnout; when nurses reported higher levels of supervisor social support they also reported higher levels of affective commitment and job satisfaction, and lower levels of anxiety and intention to leave. Others' studies have resulted in similar findings (Beehr et al., 2000; Cohen \& Wills, 1985; Ganster et al., 1986; Luchman \& González-Morales, 2013; Mayo et al., 2012; Ng \& Sorensen, 2008), including in the nursing population (Beehr et al., 1990).

Considering this study's goal is to help clarify the role of social support in moderating the stressor-strain relationship, it was first necessary to establish what the roles of social support from coworker and supervisor look like in the context of the present study. Therefore, $H_{2}$ postulated that both coworker and supervisor social support 
would moderate the relationship between stressors and outcomes, such that stressors would be less harmful for nurses reporting higher levels of social support, and more detrimental for nurses reporting lower levels of social support. This hypothesis was not supported for any of the studied interaction effects, an outcome analogous to those of Beehr et al. (2000), Ganster et al. (1986), and LaRocco et al. (1980).

If this study were to stop with results from $H_{1}$ and $H_{2}$, it might be concluded that social support has a direct effect on outcomes, but no moderating effects could be detected or it might be concluded that with a short-term longitudinal study, social support has neither ameliorative nor destructive effects on the extent to which role stressors relate to outcomes. However, such a conclusion may be misleading as it omits an understanding of how people view themselves in relation to others (i.e., self-construal), a cognitive factor that is shaped, in part, by one's cultural upbringing and thus acts as a proxy for context. In short, these results add to the litany of inconclusive results and beg for clarification by investigating what other factors may be involved in the stressorsupport-outcome process.

In order to study the extent to which self-construals affect the moderating effects of social support on the stressor-outcome relationship, $H_{3}$ and $H_{4}$ were tested. For $H_{3}$, it was expected that for nurses tending toward an interdependent self-construal, coworker social support would mitigate the effects of stressors on strains. In contrast, $H_{4}$ purported that for nurses tending toward an independent self-construal, supervisor social support would mitigate the effects of stressors on strains. In other words, the nurse's contextual framework would explain when social support from the coworker or the supervisor would 
mitigate the deleterious effects of stressors on study outcomes. Of all the stressors and outcome variables studied, it was clear that self-construal might be playing a role in the extent to which coworker support mitigates the effects of role overload (T1) on anxiety (T2), and the extent to which supervisor support mitigates the effects of role ambiguity (T1) on each of general health and burnout (T2). Moreover, these three-way effects presented when two-way effects, and/or independent effects of the expected moderator variables, did not affect the outcomes. Still, these effects did not present in the anticipated direction. Each of the three-way interaction effects is discussed below.

First, for individuals who perceived themselves as high on interdependent selfconstrual, high coworker support intensified the extent to which role overload positively related to anxiety. In contrast, for people who reported an interdependent self-construal and low coworker support the role overload-anxiety relationship was negative. For interdependents, the findings reflect a situation of reverse buffering; coworker support aggravates the extent to which role overload leads to anxiety.

As interdependents perceive themselves as closely aligned with the ingroup (Billig \& Tajfel, 1973; Tajfel, 1982), it is possible that when these nurses' workload is high and they are receiving coworker support, they may feel it as a failure to fulfill their role in the group and not contributing in alignment with the expectations. Markus and Kitayama (1991; 2010), as well as Nahum-Shani and Bamberger (2011), describe interdependents as tending more toward interactions reciprocal in nature and the idea of receiving coworker support for a situation of overload might be one example of when the coworkers' support might create a sense of "incompetence" and thus intensify anxiety. 
For interdependents in a situation of high role overload and low coworker support, the overload and limited support from coworkers might help the nurse interpret the overload as a challenge to be overcome, which would, therefore, mitigate development of anxiety.

The source congruence (or matching) hypothesis (Beehr et al., 2003; Cohen \& Wills, 1985; Dormann \& Zapf, 1999; Mayo et al., 2012; Monnot \& Beehr, 2014) might also add to the explanation of these findings. The source congruence hypothesis postulates that when the sources of occupational stressors are the same as the sources of support, it creates cognitive dissonance between the negativity of the stressors and the positivity of the support; the dissonance itself would result in additional strain. Thus, if the source of overload (whether in the form volume or difficulty of work) is the same as the source of support (i.e., the same coworkers), higher levels of coworker support might accentuate the deleterious effect of overload on anxiety for interdependent nurses. One might also consider that the time spent in supportive interactions with coworkers takes away from time otherwise needed to deal with high role overload work circumstances.

Recently, Monnot and Beehr (2014) further explored this entanglement between stressors source and support source by looking at the content of communication involved in supposedly supportive interactions and by assuming communications could be workrelated and of positive content, work-related and of negative content, or nonwork-related. The authors concluded that communications on negative topics, particularly with coworkers, had an inverse direct effect on favorable outcomes. In addition to the direct effects, it was also found that negative work-related communications intensified the harmful effects of the stressors on the outcomes, and the researchers hypothesized that, 
by engaging in these types of conversations, coworkers are likely focusing on negative aspects of the workplace and work experience and reliving angst-creating situations.

This does not exclude the assumption that interdependents have a preference for coworker support (as can be seen in the low role overload situation); perhaps in instances of high role overload, the dynamic associated with the presence of the stressor unbalances the process by which that support could have beneficial impacts, as hypothesized above. For example, Jimmieson, McKimmie, Hannam, and Gallagher (2010) explored whether team identification (including from the perspective of social identity theory; Billig \& Tajfel, 1973; Tajfel, 1982) had a higher order effect of social support as a buffer for occupational stress. Similar to the present study, theirs showed a reverse buffering effect when team identification and coworker support were high and a buffering effect in circumstances of low coworker support for those high in team identification.

As for the combined influence of role ambiguity, self-construal, and supervisor support on general health, the present study indicates that as role ambiguity increases, general health decreases slightly more for independents with high supervisor support than for independents with low supervisor support. In the case of the effect of role ambiguity, self-construal, and supervisor support on burnout, the present study indicates that as role ambiguity increases, burnout also increases for independents with high supervisor support, whereas for independents with low supervisor support burnout does not change as role ambiguity increases. However, it had been hypothesized that as role ambiguity increased, general health would have increased for independents with high supervisor support, and decreased for those with low supervisor support, whereas burnout would 
have decreased for independents with high supervisor support, and increased for those with low supervisor support. Once again, this situation is in accordance with reverse buffering.

As independents tend more toward expressing their own attributes and promoting their individual goals, maybe situations of higher supervisory support and, at the same time, greater role ambiguity, are associated with feelings of being less in control, lower self-efficacy, and greater worry (as measured by general health items), as well as increased burnout (as measured by the burnout/tedium items). For example, Lyons and Schneider (2009) showed that different leadership styles had different effects on participants' attitudes, emotions, and motivations, including self-efficacy; the transactional leadership style was associated with lower levels of self-efficacy, perceived social support, and with appraisal of stressors as more threatening than the transformational leadership style.

Perhaps independents perceived supervisory support interactions (especially if recurring over time) as an indicator of inability to face work challenges autonomously, to express and enact their goals (in this case, professional goals); this could, in turn, reinforce the experience of higher ambiguity. Conversely to a condition of high supervisor support and high role ambiguity, independents in a situation of perceived low supervisory support, and despite ambiguity, might have felt more autonomy (which is a core job characteristic that leads to high internal work motivation and job satisfaction; Hackman \& Oldham, 1980), to express their goals and, with that, express more positive thoughts and feelings as related with general health and burnout. Moreover, these 
circumstances could be approached by independents as an opportunity to overcome the ambiguity and to accomplish and further their achievements, resulting in greater levels of job control (Karasek, 1979). This interpretation would be in line with Chen and Welland's (2002) exploration of the relationship between effects of power and selfconstruals; with people in situations of power being particularly positioned for realizing their goals and with independents being motivated to promoting goals that express their unique attributes, if low support reflects a more distanced supervisor, that may create an opportunity of power, even if temporary and informal. Voyer and McIntosh (2013) further hypothesized on the relationship between power, self-construal, and leadership such that not only would independents and interdependents gravitate towards different leadership styles when in circumstances of power, but also that experiencing positions of power would influence the inclination toward an independent self-construal.

In addition to the power and self-construals perspective, and in light of these results, there is the need to consider supervisor social support may be particularly subject to the influence of frequency, nature, and content of the interaction and communication; Beehr (1995) anticipated that these elements may be responsible for much of the apparent contradiction in findings regarding the impact of social support on the occupational stress. Leadership styles reflect, to a certain extent, a pattern of interaction and communication, and a transactional leadership style (Bass, 1998, as cited in Lyons \& Schneider, 2009), characterized mainly by a process of social exchange, could potentially elicit more of the cognitions and emotions described above and also contribute to explaining present results, particularly from an independent focused on individual goal 
realization. Transformation leadership (described by inspirational motivation, idealized influence, individualized support, and intellectual stimulation), however, has been shown to be associated with reports of greater supervisory support during stressful tasks and less threat appraisals (Lee, Veasna, \& Wu, 2013; Lyons \& Schneider, 2009).

Because $H_{3}$ and $H_{4}$ were tested concomitantly, it was possible to benefit from insight into results that, despite being outside of the scope of $H_{4}$, are relevant enough to be noted in this discussion. By examining the upper graphs in Figure 7 and Figure 8, it is notable that other combinations of role stressors, social support, and self-construal do show a buffering effect. Specifically, for those tending more toward an interdependent self-construal, high supervisor support mitigated the effects of role ambiguity on general health; general health decreased more as role ambiguity increased for those with low supervisor support than for those with high supervisor support. Also, it was among those tending more toward an interdependent self-construal that high supervisor support seemed to mitigate the effects of role ambiguity on burnout; the relationship between role ambiguity and burnout was weaker for those with high supervisor support than for those with low supervisor support.

\section{Limitations and Recommendations for Future Studies}

This study has several limitations or aspects that can be addressed differently in future studies. First, it used a single composite measure of self-construal that does not approach independent and interdependent self-construals as two distinct constructs; that is, with this measure, participants had to select between an interdependent and an independent-oriented response to an item. Literature suggests self-construals work 
independently, such that one's orientation to an interdependent self-construal does not make one incapable of gravitating to an independent pattern and vice-versa (Cross et al., 2011; Oyserman, 2011; Singelis, 1994; Triandis, 1996; Voyer \& Franks, 2014). Oyserman (2011) reviewed situated cognition as the perspective associated with our natural tendency toward a self-construal or a mindset, but that in certain circumstances the less fluent mindset can be primed or activated; people who navigate in culturally diverse environments (i.e., are more culturally sensitive, such as immigrants, for example), would be more likely to show indications of interdependence and independence coexisting naturally, that is, without priming (Singelis, 1994). As such, using a measure that assesses them separately, such as Singelis' (1994), might yield greater validity and is recommended in future studies of this nature.

Second, a greater sample size as well as more heterogeneous sample might have been beneficial to a study such as this, which attempts to identify moderating effects among continuous variables. Viswesvaran et al. (1999), in their influential meta-analysis on the role of social support in occupational stress, as well as Ganster et al. (1986), noted that numerous studies employing moderated regression analyses are constrained by samples that are relatively small for the number of variables that are being analyzed. A larger sample size will increase the statistical power to test three-way interactions as in the current study. Concurrently, the level of homogeneity of the sample may also be prejudicial, given that the sample is exclusively nurses and also overwhelmingly female. Not only does this sample constraint not allow for a more robust testing of third order interactions effects, but it also reduces the ability to generalize findings, with results only 
being interpretable within a limited set of circumstances. For example, Ganster et al. (1986) discussed how sex may be involved in moderating the effect of social support on the relationship between stressors and outcomes at work; women and men showed different types of supportive relationships and for women, non-work sources of support moderated the work stress-burnout relation, and for men, work sources of support did. More recently, Voyer and Reader (2013) showed significant differences between physicians and nurses in their levels of independent self-construal (physicians showed higher levels than nurses) and in their levels of interdependent self-construal when sex was taken into account (male nurses and female physicians showed higher levels of interdependent self-construal than female nurses and male physicians). Still, constraining the population type is an advantage in that the findings are likely relevant to that population specifically and help to reduce the complexity of the already complex model.

Third, even though the current study was of a longitudinal nature, thereby addressing limitations identified by previous studies (Beehr et al., 2003; Mayo et al., 2012), future longitudinal studies may benefit from also tracking and controlling for any work-related events, such as organizational and leadership changes, that may happen between Time 1 and Time 2. For example, if participants' supervisors or teams changed from Time 1 to Time 2, those events themselves can influence their responses.

Fourth, from an analytical perspective, future research can explore alternative analytical methodologies that identify non-linear effects. What if the effects of supervisor and coworker social support are better represented by a model in which a moderate "amount" of social support is optimal in buffering strain, rather than a linear 
model that purports a "the-more-the-better" effect? This type of relationship has been seen with other constructs considered to be job resources; Kubicek, Korunka, and Tement (2014) showed that for eldercare nurses, high and low levels of job control were associated with higher levels of unfavorable outcomes and lower levels of favorable outcomes, with the reverse being observed for nurses with medium levels of job control. Similarly, Hesselgraves and Scholarios (2014), and before them Harris and Kacmar (2006), observed indications, in some types of roles, of a curvilinear relationship between leader-member exchange (LMX) and strains such that low and high levels of LMX were associated with higher levels of strain and moderate levels of LMX with lower levels of strain comparatively.

Finally, recalling the Beehr and Newman $(1995 ; 1998)$ facet model, and in light of continuing mixed results, such as those of this and other studies, future investigations should broaden the current paradigm to consider new perspectives as discussed below (Beehr, 1995). These studies continue to point towards a paradigm where social support as a contributing factor (as per the situational characteristics facet; see Figure 1), may be dependent upon other interacting circumstances, as hypothesized earlier while discussing current results; these would include other situational characteristics (which could also act as primes), personal characteristics like self-construals and others. This dynamic would then result in circumstances where different types of social support would sometimes present a mitigating effect, sometimes present an intensifying effect, and sometimes not present a significant effect at all; all of these were seen in the current study. As mentioned earlier, Beehr (1995) discussed aspects of social support, such as content of 
communication, as well as frequency and nature of interactions, which could be assessed in future studies. Studying these aspects of social support would also allow for deeper analyses into hypotheses that have had some research, such as the source congruence hypothesis (Beehr et al., 2003; Mayo et al., 2012). Aside from a different approach into how social support can impact the occupational stress process from a situational characteristics facet perspective, Beehr (1995) also called for broadening research into considering that social support can also be a dependent variable, an effect, and not just a cause; that the recipient of support is not just passively receiving support and can also actively look for it which, from a theory perspective, would position social support not just in the situational characteristics facet but also in the coping and adaptation facet. If supervisor and coworker social support are procured by employees in situations of high levels of strain caused by high levels of stressors and, if independent- and interdependentprone employees procured that support differently in quantity and/or quality, then it is conceivable that higher levels of reported social support may result from stronger relationships between stressors and outcomes, and differently so for independents and interdependents.

\section{Conclusion}

This study contributed to the literature on the role of social support in occupational stress literature by adding to the growing body of evidence that shows the importance of context in identifying and clarifying what that role may be. That there were significant three-way interactions between role stressor, source of social support, and self-construal, but no two-way interactions between any two of the above set of 
variables, reflects the importance of context. This study showed there can be situations when there are no moderating effects of social support or self-construal on the stressoroutcome relationship, but there can be buffering effects when both support and selfconstrual are considered, thus illustrating the complexity underlying the role of social support in occupational stress. These complex results reinforce the need to continue to explore multifaceted models. Even the social support construct is complex and this study shows the need to discriminate between different types of social support. The current study also replicated the commonly found direct effects of social support on outcomes.

With company management and human resources implementing interventions targeted at leveraging social support to mitigate occupational stress, it is increasingly important that those interventions are adapted to take into account context. From the direct effects results it can be seen that social support interventions targeted at reducing certain strains are helpful. However, if research demonstrates that for employees with certain personal characteristics, in face of certain deleterious work stressors, more coworker or supervisor support will not necessarily curb the effects of those harmful stressors have on outcomes, then interventions need to target or create the context where more support does in fact produce that mitigation. 


\section{References}

Abdel-Halim, A. (1978). Employee affective responses to organizational stress: Moderating effects of job characteristics. Personnel Psychology, 31, 561-579. doi:10.1111/j.1744-6570.1978.tb00463.x

Aiken, L. S., \& West, S. G. (1991). Multiple regression: Testing and interpreting interactions. Newbury Park, CA: Sage.

Allen, N. J., \& Meyer, J. P. (1990). The measurement and antecedents of affective, continuance and normative commitment to the organization. Journal of Occupational Psychology, 63, 1-18.

American Institute of Stress. (n.d.). Job Stress. Retrieved March 11, 2012, from American Institute of Stress: http://www.stress.org/job.htm

American Psychological Association. (2015, February 4). Stress in America Press Room, 2014. Retrieved March 11, 2015, from American Psychological Association: http://www.apa.org/news/press/releases/stress/

Banks, M. H., Clegg, C. W., Jackson, P. R., Kemp, N. J., Stafford, E. M., \& Wall, T. D. (1980). The use of the General Health Questionnaire as an indicator of mental health in occupational studies. Journal of Occupational Psychology, 53, 187-194.

Beehr, T. A. (1995). Psychological stress in the workplace. New York: Routledge.

Beehr, T. A. (1998). An organizational psychology meta-model of occupational stress. In C. L. Cooper (Ed.), Theories of organizational stress (pp. 6-27). New York: Oxford University Press.

Beehr, T. A., \& Glazer, S. (2001). A cultural perspective of social support in relation to occupational stress. In P. Perrewé, \& D. C. Ganster (Eds.), Research in occupational stress and well-being, Vol. 1. Exploring theoretical mechanisms and perspectives (pp. 97-142). Greenwich, CO: JAI Press Inc.

Beehr, T. A., \& Glazer, S. (2005). Organizational role stress. In J. Barling, E. K. Kelloway, \& M. R. Frone (Eds.), Handbook of work stress (pp. 7-33). Thousand Oaks, CA: Sage.

Beehr, T. A., \& Newman, J. E. (1978). Job stress, employee health, and organizational effectiveness: A facet analysis, model and literature review. Personnel Psychology, 31, 665-699. 
Beehr, T. A., Bowling, N. A., \& Bennett, M. M. (2010). Occupational stress and failures of social support: When helping hurts. Journal of Occupational Health

Psychology, 15, 45-59. doi:10.1037/a0018234

Beehr, T. A., Farmer, S. J., Glazer, S., Gudanowski, D. M., \& Nair, V. N. (2003). The enigma of social support and occupational stress: Source congruence and gender role effects. Journal of Occupational Health Psychology, 8, 220-231.

Beehr, T. A., Jex, S. M., Stacy, B. A., \& Murray, M. A. (2000). Work stressors and coworker support as predictors of individual strain and job performance. Journal of Organizational Behavior, 21, 391-405.

Beehr, T. A., King, L. A., \& King, D. W. (1990). Social support and occupational stress: Talking to supervisors. Journal of Vocational Behavior, 36, 61-81.

Beehr, T. A., Walsh, J. T., \& Taber, T. D. (1976). Relationship of stress to individually and organizationally valued states: Higher order needs as a moderator. Journal of Applied Psychology, 61, 41-47. doi:10.1037/0021-9010.61.1.41

Billig, M., \& Tajfel, H. (1973). Social categorization and similarity in intergroup behaviour. European Journal of Social Psychology, 3, 27-52.

Bureau of Labor Statistics. (2012, March 29). Occupational Outlook Handbook, 20122013 Edition, Registered Nurses. Retrieved April 20, 2012, from Bureau of Labor Statistics, U.S. Department of Labor:

http://www.bls.gov/ooh/healthcare/registered-nurses.htm\#tab-4

Cammann, C., Fichman, M., Jenkins, Jr., G. D., \& Klesh, J. R. (1983). Assessing the attitudes and perceptions of organizational members. In S. E. Seashore, E. E. Lawler III, P. H. Mirvis, \& C. Cammann (Eds.), Assessing organizational change (pp. 71-138). New York: Wiley.

Caplan, R. D., Cobb, S., French, J., Harrison, R. V., \& Pinneau, S. R. (1975). Job demands and worker health: Main effects and occupational differences. Washington DC: US Government Printing Office.

Chen, S., \& Welland, J. (2002). Examining the effects of power as a function of selfconstruals and gender. Self and Identity, 1, 251-269. doi:10.1080/15298860290063768

Cohen, S., \& Wills, T. A. (1985). Stress, social support, and the buffering hypothesis. Psychological Bulletin, 98, 310-357. 
Cross, S. E., Hardin, E. E., \& Gercek-Swing, B. (2011). The what, how, why and where of self-construal. Personality and Social Psychology Review, 15, 142-179. doi:10.1177/1088868310373752

Da Silva, N., \& Oldham, G. R. (2012). Adopting employees' ideas: Moderators of the idea generation - idea implementation link. Creativity Research Journal, 24(2-3), 134-145. doi:10.1080/10400419.2012.677257

Da Silva, N., Hutcheson, J., \& Wahl, G. D. (2010). Organizational strategy and employee outcomes: A person-organization fit perspective. The Journal of Psychology, 144, 145-161.

Damrosch, S. P. (1986). Ensuring anonymity by use of subject-generated identification codes. Research in Nursing \& Health, 9, 61-63.

Dawson, J. F. (2014). Moderation in management research: What, why, when and how. Journal of Business and Psychology, 29, 1-19. doi:10.1007/s10869-013-9308-7

DiIorio, C., Soet, J. E., Van Marter, D., Woodring, T. M., \& Dudley, W. N. (2000). An evaluation of a self-generated identification code. Research in Nursing \& Health, $23,167-174$.

Dormann, C., \& Zapf, D. (1999). Social support, social stressors at work, and depressive symptoms: testing for main and moderating effects with structural equations in a three-wave longitudinal study. Journal of Applied Psychology, 84, 874-884.

Edwards, J. R., \& Harrison, R. V. (1993). Job demands and worker health: Threedimensional reexamination of the relationship between person-environment fit and strain. Journal of Applied Psychology, 78, 628-648.

Fischer, R., Ferreira, M. C., Assmar, M. C., Redford, P., Harb, C., Glazer, S., . . Achoui, M. (2009). Individualism-collectivism as descriptive norms: Development of a subjective norm approach to cultural measurement. Journal of Cross-Cultural Psychology, 40, 187-213.

French, J. R., Caplan, R. D., \& Harrison, R. V. (1982). The mechanisms of job stress and strain. New York: Wiley.

Ganster, D. C., Fusilier, M. R., \& Mayes, B. T. (1986). Role of social support in the experience of stress at work. Journal of Applied Psychology, 71, 102-110.

Glazer, S. (2005). Six of one, half a dozen of the other: Fixed versus rotating shifts might not really matter when it comes to work-related variables. International Journal of Stress Management, 12, 142-163. 
Glazer, S. (2006). Social support across cultures. International Journal of Intercultural Relations, 30, 605-622.

Glazer, S., \& Beehr, T. A. (2005). Consistency of the implications of three role stressors across four countries. Journal of Organizational Behavior, 26, pp. 467-487.

Glazer, S., \& Gyurak, A. (2008). Sources of occupational stress among nurses in five countries. International Journal of Intercultural Relations, 32, 49-66.

Goldberg, D. P., \& Hillier, V. F. (1979). A scaled version of the General Health Questionnaire. Psychological Medicine, 9, 139-145.

Goldberg, D. P., Gater, R., Sartorius, N., Ustun, T. B., Piccinelli, M., Gureje, O., \& Rutter, C. (1997). The validity of two versions of the GHQ in the WHO study of mental illness in general health care. Psychological Medicine, 27, 191-197.

Goldstein, S. (2008). Activity 5.1: The interdependent and independent selves. In Crosscultural explorations: Activities in culture and psychology (2nd ed.). Boston, MA: Pearson.

Hackman, J. R., \& Oldham, G. R. (1980). Work Redesign. Reading, MA: AddisonWesley.

Harris, K. J., \& Kacmar, K. M. (2006). Too much of a good thing: The curvilinear effect of leader-member exchange on stress. The Journal of Social Psychology, 146, 6584 .

Harrison, R. V. (1978). Person-Environment fit and job stress. In C. Cooper, \& R. Payne (Eds.), Stress at work (pp. 175-206). New York: Wiley.

Health and Safety Executive. (n.d.). Statistics: Stress-related and psychological disorders in Great Britain. Retrieved March 11, 2012, from Health and Safety Executive: http://www.hse.gov.uk/statistics/causdis/stress/index.htm

Hesselgraves, H., \& Scholarios, D. (2014). Leader-member exchange and strain: A study of job demands and role status. Human Resource Management Journal, 24, 459478. doi:10.1111/1748-8583.12042

House, J. S. (1981). Work stress and social support. Reading, MA: Addison-Wesley.

HumanNature@ Work. (2008). Stats \& Quotes: Facts and Figures about Stress in the Workplace. Retrieved March 11, 2012, from HumanNature@ Work: http://www.humannatureatwork.com/serious.htm

Jex, S. M. (2002). Organizational psychology: A scientist-practitioner approach. New York: Wiley. 
Jex, S. M., \& Beehr, T. A. (1991). Emerging theoretical and methodological issues in the study of work-related stress. Research in Personnel and Human Resources Management, 9, 311-365.

Jex, S. M., Beehr, T. A., \& Roberts, C. K. (1992). The meaning of occupational stress items to survey respondents. Journal of Applied Psychology, 77, 623-628.

Jimmieson, N. L., McKimmie, B. M., Hannam, R. L., \& Gallagher, J. (2010). An investigation of the stress-buffering effets of social support in the occupational stress process as a function of team identification. Group Dynamics: Theory, Research, and Practice, 14, 350-367. doi:10.1037/a0018631

Kahn, R. L., Wolfe, D. M., Quinn, R. P., Snoek, J. D., \& Rosenthal, R. A. (1964). Organizational stress: Studies in role conflict and ambiguity. Oxford, England: Wiley.

Karasek, R. A. (1979). Job demands, job decision latitude, and mental strain: Implications for job redesign. Administrative Science Quarterly, 24, 285-308.

Katz, D., \& Kahn, R. L. (1978). The social psychology of organizations (2nd ed.). New York: Wiley.

Kaufmann, G. M., \& Beehr, T. A. (1986). Interactions between job stressors and social support: Some counterintuitive results. Journal of Applied Psychology, 71, 522526.

King, L. A., \& King, D. W. (1990). Role conflict and role ambiguity: A critical assessment of construct validity. Psychological Bulletin, 107, 48-64.

Kivimäki, M., Hotopf, M., \& Henderson, M. (2010). Do stressful working conditions cause psychiatric disorders? Occupational Medicine, 60, 86-87.

Kubicek, B., Korunka, C., \& Tement, S. (2014). Too much job control? Two studies on curvilinear relations between job control and eldercare workers' well-being. International Journal of Nursing Studies, 51, 1644-1653.

LaRocco, J. M., House, J. S., \& French, J. R. (1980). Social support, occupational stress, and health. Journal of Health and Social Behavior, 21, 202-218.

Lazarus, R. S., \& Folkman, S. (1984). Stress, appraisal and coping. New York: Springer.

Lee, L.-Y., Veasna, S., \& Wu, W.-Y. (2013). The effects of social support and transformational leadership on expatriate adjustment and performance. Career Development International, 18, 377-415. doi:10.1108/CDI-06-2012-0062 
Luchman, J. N., \& González-Morales, M. G. (2013). Demands, control, and support: A meta-analytic review of work characteristics interrelationships. Journal of Occupational Health Psychology, 18, 37-52. doi:10.1037/a0030541

Lyons, J. B., \& Schneider, T. R. (2009). The effects of leadership style on stress outcomes. The Leadership Quarterly, 20, 737-748.

doi:10.1016/j.leaqua.2009.06.010

Malach-Pines, A. (2005). The burnout measure, short version. International Journal of Stress Management, 12, 78-88. doi:10.1037/1072-5245.12.1.78

Markus, H. R., \& Kitayama, S. (1991). Culture and the self: Implications for cognition, emotion and motivation. Psychological Review, 98, 224-253.

Markus, H. R., \& Kitayama, S. (2010). Cultures and selves: A cycle of mutual constitution. Perspectives on Psychological Science, 5, 420-430. doi:10.1177/1745691610375557

Mayo, M., Sanchez, J. I., Pastor, J. C., \& Rodriguez, A. (2012). Supervisor and coworker support: A source congruence approach to buffering role conflict and physical stressors. International Journal of Human Resource Management, 23, 3872-3889. doi:10.1080/09585192.2012.676930

McClelland, D. C. (1961). The Achieving Society. Princeton, NJ: Van Nostrand.

McClelland, D. C. (1988). Human Motivation. Cambridge: Cambridge University Press.

McGrath, J. E. (1976). Stress and behavior in organizations. In M. D. Dunnette (Ed.), Handbook of industrial and organizational psychology (pp. 1351-1396). Chicago, IL: Rand McNally College Publishing Company.

Monnot, M. J., \& Beehr, T. A. (2014). Subjective well-being at work: Disentangling source effects of stress and support on enthusiasm, contentment, and meaningfulness. Journal of Vocational Behavior, 85, 204-218.

Morimoto, H., Shimada, H., \& Ozaki, K. (2013). Does stressor evaluation mediate sociocultural influence on coping selection? An investigation using japanese employees. International Journal of Stress Management, 20, 1-19. doi:10.1037/a0031432

Munro, L., Rodwell, J., \& Harding, L. (1998). Assessing occupational stress in psychiatric nurses using the full job strain model: The value of social support to nurses. International Journal of Nursing Studies, 35, 339-345. 
Nahum-Shani, I., \& Bamberger, P. A. (2011). Explaining the variable effects of social support on work-based stressor-strain relations: The role of perceived pattern of support exchange. Organizational Behavior and Human Decision Processes, 114, 49-63.

National Institute for Occupational Safety and Health. (1999). NIOSH-Publications and Products: Stress... at Work. Retrieved March 11, 2012, from CDC - NIOSH: http://www.cdc.gov/niosh/docs/99-101/pdfs/99-101.pdf

National Institute for Occupational Safety and Health. (2008, July). Exposure to Stress: Occupational Hazards in Hospitals. Retrieved June 2013, from CDC - NIOSH: http://www.cdc.gov/niosh/docs/2008-136/pdfs/2008-136.pdf

Nezlek, J. B., Kafetsios, K., \& Smith, C. V. (2008). Emotions in everyday social encounters: Correspondence between culture and self-construal. Journal of CrossCultural Psychology, 39, 366-372. doi:10.1177/0022022108318114

Ng, T. W., \& Sorensen, K. L. (2008). Toward a further understanding of the relationships between perceptions of support and work attitudes. Group \& Organization Management, 33, 243-268. doi:10.1177/1059601107313307

O.Net. (2010). Summary Report for: 29-1141.00 - Registered Nurses. Retrieved March 11, 2012, from O.Net OnLine: http://www.onetonline.org/link/summary/291141.00

O'Driscoll, M. P., \& Dewe, P. J. (2001). Mediators and moderators of stressor-strain linkages. In P. L. Perrewé, \& D. C. Ganster (Eds.), Research in occupational stress and well-being: Vol. 1. Exploring theoretical mechanisms and perspectives (pp. 257-287). Greenwich, CO: JAI Press Inc.

Oyserman, D. (2011). Culture as situated cognition: Cultural mindsets, cultural fluency, and meaning making. European Review of Social Psychology, 22, 164-214. doi:10.1080/10463283.2011.627187

Parker, D. F., \& DeCotiis, T. A. (1983). Organizational determinants of job stress. Organizational Behavior and Human Performance, 32, 160-177.

Peters, L. H., \& O'Connor, E. J. (1980). Situational constraints and work outcomes: The influences of a frequently overlooked construct. Academy of Management Review, 5, 391-397.

Rizzo, J. R., House, R. J., \& Lirtzman, S. I. (1970). Role conflict and ambiguity in complex organizations. Administrative Science Quarterly, 15, 150-163. Retrieved from http://www.jstor.org/stable/2391486 
Schwartz, S. H. (1999). A theory of cultural values and some implications for work. Applied Psychology: An International Review, 48, 23-47.

Semmer, N. K., Elfering, A., Jacobshagen, N., Perrot, T., Beehr, T. A., \& Boos, N. (2008). The emotional meaning of instrumental social support. International Journal of Stress Management, 15, 235-251.

Singelis, T. M. (1994). The measurement of independent and interdependent selfconstruals. Personality and Social Psychology Bulletin, 20, 580-591.

Sonnentag, S., \& Frese, M. (2003). Stress in organizations. In W. C. Borman, D. R. Ilgen, \& R. J. Klimoski (Eds.), Handbook of Psychology, Vol. 12. Industrial and organizational psychology (pp. 453-491). Hoboken, NJ: Wiley.

Spetz, J., Keane, D., \& Herrera, C. (2011). 2010 Survey of Registered Nurses. University of California, San Francisco, School of Nursing. Sacramento, CA: California Board of Registered Nursing.

Tajfel, H. (1982). Social psychology of intergroup relations. Annual Review of Psychology, 33, 1-39.

Toivanen, S. (2012). Social determinants of stroke as related to stress at work among working women: A literature review. Stroke Research and Treatment, 2012, 1-10. doi:10.1155/2012/873678

Triandis, H. C. (1996). The psychological measurement of cultural syndromes. American Psychologist, 51, 407-415.

Tsutsumi, A., Kayaba, K., \& Ishikawa, S. (2011). Impact of occupational stress on stroke across occupational classes and genders. Social Science \& Medicine, 72, 16521658. doi:10.1016/j.socscimed.2011.03.026

Tyler, P., \& Cushway, D. (1995). Stress in nurses: The effects of coping and social support. Stress Medicine, 11, 243-251.

Van Der Doef, M., \& Maes, S. (1999). The Job Demand-Control(-Support) Model and psychological well-being: a review of 20 years of empirical research. Work and Stress, 13, 87-114.

Viswesvaran, C., Sanchez, J. I., \& Fisher, J. (1999). The role of social support in the process of work stress: A meta-analysis. Journal of Vocational Behavior, 54, 314334. 
Voyer, B. G., \& Franks, B. (2014). Toward a better understanding of self-construal theory: An agency view of the processes of self-construal. Review of General Psychology, 18, 101-114. doi:10.1037/gpr0000003

Voyer, B. G., \& McIntosh, B. (2013). The psychological consequences of power and selfperception: Implications for leadership. Leadership \& Organization Development Journal, 34, 639-660. doi:10.1108/LODJ-10-2011-0104

Voyer, B. G., \& Reader, T. (2013). The self-construal of nurses and doctors; Beliefs on interdependence and independence in the care of older people. Journal of Advanced Nursing, 69, 2696-2706. doi:10.1111/jan.1215

Wanous, J. P., \& Reichers, A. E. (1996). Estimating the reliability of a single-item measure. Psychological Reports, 78, 631-634.

Wanous, J. P., Reichers, A. E., \& Hudy, M. J. (1997). Overall job satisfaction: How good are single-item measures? Journal of Applied Psychology, 82, 247-252.

Wu, H., Chi, T.-S., Chen, L., Wang, L., \& Jin, Y.-P. (2010). Occupational stress among hospital nurses: Cross-sectional survey. Journal of Advanced Nursing, 66, 627634.

Yurek, L. A., Vasey, J., \& Havens, D. S. (2008). The use of self-generated identification codes in longitudinal research. Evaluation Review, 32, 435-452. doi:10.1177/0193841X08316676

Zapf, D., Dormann, C., \& Frese, M. (1996). Longitudinal studies in organizational stress research: A review of the literature with reference to methodological issues. Journal of Occupational Health Psychology, 1, 145-169. 


\section{Appendix A - List of Items Used for Current Study}

\section{Role Stressors (Time 1)}

Role Overload:

1. I receive an assignment without the manpower to complete it

2. It seems like I have too much work for one person to do

3. On my present job, the amount of work seems to interfere with how well I can do the job

4. I often notice a marked increase in my workload

Role Conflict:

5. I work with two or more groups who operate quite differently

6. I receive incompatible requests from two or more people

7. I do things that are apt to be accepted by one person and not accepted by another

Role Ambiguity:

8. I have clear, planned goals and objectives for my job (R)

9. I know exactly what is expected of me (R)

10. Explanation is clear of what has to be done $(\mathrm{R})$ 


\section{Moderators}

Social Support (Supervisor; Time 1)

11. My supervisor at work goes out of his or her way to do things to make my work life easier for me

12. It is easy to talk with my supervisor at work

13. I can rely on my supervisor at work when things get tough at work

14. My supervisor at work is willing to listen to my personal problems

Social Support (Coworker; Time 1)

15. Other people at work go out of their way to do things to make my work life easier for me

16. It is easy to talk with other people at work

17. I can rely upon other people at work when things get tough at work

18. Other people at work are willing to listen to my personal problems

Self-Construals (Time 2)

19. Success depends on help from others / Success depends on my abilities

20. I know more about others than I do about myself / I know more about myself than I do about others

21. Being excluded from my group would be very hard on me / Being dependent on others would be very hard on me

22. Silence is comfortable / Silence is embarrassing

23. It is important that my behavior is appropriate for the situation / It is important that my behavior and attitudes correspond

24. I sometimes feel ashamed / I sometimes feel guilty

25. Friendships are difficult to establish, but are generally very intimate / Friendships are fairly easy to establish, but often not very intimate 


\section{Strains/Outcomes (Time 2)}

Job-Related Anxiety:

26. Sometimes when I think about my job I get a tight feeling in my chest

27. I have felt fidgety or nervous as a result of my job

28. My job gets to me more than it should

29. There are lots of times when my job drives me right up the wall

\section{Affective Commitment:}

30. This organization has a great deal of personal meaning for me

31. I do not feel a strong sense of belonging to my organization (R)

32. I do not feel "emotionally attached" to this organization (R)

33. I do not feel like "part of the family" at my organization (R)

34. I would be very happy to spend the rest of my career with this organization

35. I enjoy discussing my organization with people outside of it

36. I really feel as if this organization's problems are my own

37. I think that I could easily become as attached to another organization as I am to this one $(\mathrm{R})$

Intention to Leave:

38. I will actively look for a new job in the next year

39. I often think about quitting

40. I will probably look for a new job in the next year

\section{General Health:}

41. I have been able to concentrate on what I am doing

42. I have lost much sleep over worry (R) 
43. I have felt that I am playing a useful part in things

44. I have felt capable of making decisions about things

45. I have felt I can't overcome my difficulties (R)

46. I have been able to enjoy my normal day-to-day activities

47. I have been able to face up to my problems

48. I have been feeling unhappy or depressed (R)

49. I have been losing confidence in myself $(\mathrm{R})$

50. I have been thinking of myself as a worthless person (R)

51. I have been feeling reasonably happy, all things considered

Job Satisfaction:

52. Overall, I am satisfied working at this organization

Burnout ("When you think about your work overall, how often do you feel the following?"):

53. Tired

54. Disappointed with people

55. Hopeless

56. Trapped

57. Helpless

58. Depressed

59. Physically weak / Sickly

60. Worthless / Like a failure

61. Difficulties sleeping

62. "I've had it" 


\section{Self-Generated Identification Code Questions}

63. So that we may match this survey to the next survey, please provide your parents' initials, starting with your father (or male caretaker/role model) then your mother's (or female caretaker/role model), e.g., Jeffrey Michael Smith and Sandra Costas (maiden name please) will be: JMSSC.

64. What is the first letter of the name of your elementary school (e.g., Summit Park $=>$ S)?

65. What is the last digit of the year you graduated high school (e.g., $1976=>6$ )?

66. Were you born in an even-numbered year? Yes No

67. How many older siblings (living and deceased; please include half-siblings) do you have? 